Pure and Applied Mathematics Quarterly

Volume 10, Number 1

(Special Issue: In memory of

Andrey Todorov, Part 2 of 3)

$155-192,2014$

\title{
The Space of Complete Quotients
}

\author{
Yi Hu and Yijun Shao
}

\begin{abstract}
We introduce complete quotients over the projective line and prove that they form smooth projective varieties. The resulting parameter spaces coincide with the varieties constructed in [HLS11] and [Shao11]. Hence they provide modular smooth compactifications with normal crossing boundaries of the spaces of algebraic maps from the projective line to Grassmannian varieties, resolving the singularities of the boundaries of the Quot scheme compactifications.
\end{abstract}

Keywords: Quot scheme, Complete Quotient.

\section{INTRODUCTION}

We work throughout over an algebraically closed field $\mathbb{k}$ of characteristic 0 .

Fix the Grassmannian $\operatorname{Gr}(k, V)$ of $k$-dimensional subspace in a vector space $V \cong \mathbb{k}^{n}$. The set $\operatorname{Mor}_{d}\left(\mathbb{P}^{1}, \operatorname{Gr}(k, V)\right)$ of degree $d$ algebraic maps from $\mathbb{P}^{1}$ to $\operatorname{Gr}(k, V)$ has a natural structure of a smooth quasi-projective variety. It comes with a natural compactification, the Grothendieck Quot scheme $Q_{d}:=\operatorname{Quot}_{V_{\mathbb{P}^{1}} / \mathbb{P}^{1} / \mathbb{k}}^{d, n-k}$, parametrizing all equivalence classes $\left[V_{\mathbb{P}^{1}} \rightarrow F\right]$ of quotients of degree $d$ and rank $n-k$, where $V_{\mathbb{P}^{1}}:=V \otimes_{\mathbb{k}} \mathcal{O}_{\mathbb{P}^{1}}$. The subset

$$
\stackrel{\circ}{Q}_{d}:=\left\{\left[V_{\mathbb{P}^{1}} \rightarrow F\right] \in Q_{d} \mid F \text { is locally free }\right\}
$$

is open in $Q_{d}$ and can be identified with the variety $\operatorname{Mor}_{d}\left(\mathbb{P}^{1}, \operatorname{Gr}(k, V)\right)$.

Received February 1, 2013. 
The compactification $Q_{d}$ is smooth but the boundary $Q_{d} \backslash \stackrel{\circ}{Q}_{d}$ has rather intricate singularities. It comes equipped with a natural filtration by closed subsets

$$
Z_{d, 0} \subset Z_{d, 1} \subset \cdots \subset Z_{d, d-1}=Q_{d} \backslash \stackrel{\circ}{Q}_{d}
$$

where $Z_{d, r}=\left\{\left[V_{\mathbb{P}^{1}} \rightarrow F\right] \in Q_{d} \mid\right.$ the torsion of $F$ has degree $\left.\geq d-r\right\}, r=$ $0, \ldots, d-1$. The subsets $Z_{d, r}$ admit natural subscheme structures (cf. Section 3, [Shao11]).

Theorem 1.1 ([HLS11, Shao11]). The singularities of the subscheme $Z_{d, r}$ can be resolved by repeatedly blowing up $Z_{d, 0}, Z_{d, 1}, \cdots, Z_{d, r-1}$. Consequently, by iteratively blowing-up the Quot scheme $Q_{d}$ along $Z_{d, 0}, Z_{d, 1}, \cdots, Z_{d, d-1}$, we obtain a smooth compactification $\widetilde{Q}_{d}$ such that the boundary $\widetilde{Q}_{d} \backslash \stackrel{\circ}{Q}_{d}$ is a simple normal crossing divisor.

The authors of [HLS11] proved the case when $k=1$. In his Ph.D thesis [Shao11], the second named author proved it for all Grassmannians $\operatorname{Gr}(k, V)$, $k \geq 1$.

The main purpose of this paper is to provide $\widetilde{Q}_{d}$ the following modular interpretation.

For any coherent sheaf $X$ over $\mathbb{P}^{1}$, we let $X^{\mathbf{t}}$ denote the torsion subsheaf of $X$ and $X^{\mathbf{f}}=X / X^{\mathbf{t}}$ denote the free part of $X$. For any coherent sheaves $A$ and $B$ on $\mathbb{P}^{1}$, an extension of $A$ by $B$ is a short exact sequence $0 \rightarrow B \rightarrow X \rightarrow A \rightarrow 0$, which will also be shorthanded by $B \longmapsto X \rightarrow A$. Two extensions $B \longmapsto X_{1} \rightarrow A$ and $B \longmapsto X_{2} \rightarrow A$ differ by a scalar multiple $\lambda$ if there is an isomorphism $X_{1} \simeq X_{2}$ such that the following diagram

$$
\begin{aligned}
& 0 \longrightarrow B \longrightarrow X_{1} \longrightarrow A \longrightarrow 0 \\
& \lambda \cdot \downarrow \quad \downarrow \simeq \| \\
& 0 \longrightarrow B \longrightarrow X_{2} \longrightarrow A \longrightarrow 0
\end{aligned}
$$

commutes, where $\lambda$. stands for the multiplication by the scalar $\lambda$. We use $[B \longmapsto$ $X \rightarrow A]$ to denote the equivalence class of the extension $B \longmapsto X \rightarrow A$ modulo scalar multiplication.

Definition 1.2. A complete quotient of $V_{\mathbb{P}^{1}}$ of degree $d$ and rank $n-k$ on $\mathbb{P}^{1}$ is either

- a quotient $\left[V_{\mathbb{P} 1} \rightarrow X_{1}\right] \in Q_{d}$ such that $X_{1}$ is locally free; or, 
- a sequence $\left(\left[V_{\mathbb{P}^{1}} \rightarrow X_{1}\right],\left[X_{1}^{\mathbf{f}} \longmapsto X_{2} \rightarrow X_{1}^{\mathbf{t}}\right], \ldots,\left[X_{m}^{\mathbf{f}} \longmapsto X_{m+1} \rightarrow X_{m}^{\mathbf{t}}\right]\right)$ with $m \geq 1$ such that $\left[V_{\mathbb{P} 1} \rightarrow X_{1}\right] \in Q_{d}$, for every $1 \leq i \leq m, X_{i+1}$ is a non-split extension of $X_{i}^{\mathbf{t}}$ by $X_{i}^{\mathbf{f}}$, and further, the last sheaf $X_{m+1}$ is the unique one that is locally free.

It follows from the definition that all the sheaves $X_{1}, \ldots, X_{m+1}$ are coherent and have degree $d$ and rank $n-k$.

The main theorem of this paper is

Theorem 1.3. The projective variety $\widetilde{Q}_{d}$ parameterizes all complete quotients of $V_{\mathbb{P}^{1}}$ of degree $d$ and rank $n-k$.

To prove this theorem, we calculate the normal bundle of the blowup center in each blowup of $\widetilde{\mathcal{Q}}_{d} \longrightarrow Q_{d}$ as presented in [HLS11, Shao11], and modularly interpret the points in the normal bundle as the desired extensions.

This work is the genus zero case of a larger project. The higher genus case will appear in forthcoming papers.

Acknowledgement. While this paper being prepared, the first named author was partially supported by NSF DMS 0901136. He would also like to dedicate this work to the memory of Andrey Todorov who passed away in Jerusalem in the spring of 2012 .

The notations in this paper closely follow those in [Shao11] with occasional modifications.

\section{The SPACE OF RELATIVE EXTENSIONS}

2.1. The space of non-split extensions. Let $F$ and $T$ be two coherent sheaves over $\mathbb{P}^{1}$ with $F$ being locally free and $T$ torsion. The vector space $E=\operatorname{Ext}_{\mathbb{P}^{1}}^{1}(T, F)$ can be identified with the set of all (isomorphism classes of) extensions $F \longmapsto$ $X \rightarrow T$ of $T$ by $F$. The zero element corresponds to the split extension $F \longmapsto$ $F \oplus T \rightarrow T$ while the remainders correspond to non-split ones. Therefore the projective space $\mathbb{P}(E):=\operatorname{Proj}\left(\operatorname{Sym}^{*}\left(E^{\vee}\right)\right)$, where $E^{\vee}$ is the linear dual of the vector space $E$, parametrizes the set of all non-split extensions of $T$ by $F$ up to scalar multiplication. Here again, as in the introduction, two extensions $F \longmapsto$ 
$X_{1} \rightarrow T$ and $F \longmapsto X_{2} \rightarrow T$ differ by a nonzero scalar multiple $\lambda$ if and only if there is an isomorphism $X_{1} \simeq X_{2}$ that makes the following diagram commute:

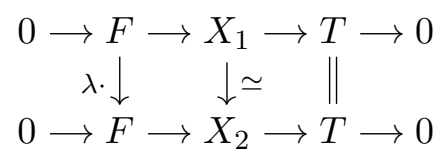

where $\lambda$. is the scalar multiplication by $\lambda$. We denote by $[F \longmapsto X \rightarrow T]$ the point of $\mathbb{P}(E)$ corresponding to a non-split extension $F \longmapsto X \rightarrow T$. One checks that an extension $F \longmapsto X \rightarrow T$ splits if and only if $\operatorname{deg} X^{\mathbf{t}}=\operatorname{deg} T$. For a discussion on universal extensions, see Example 2.1.12, [HL97] .

We need to introduce a relative version of the space of non-split extensions. For this, we begin with a lemma.

Lemma 2.1. Let $S$ be a noetherian scheme, $0 \rightarrow \mathcal{E}_{1} \rightarrow \mathcal{E}_{0} \rightarrow \mathcal{T} \rightarrow 0$ a short exact sequence of coherent sheaves on $S$ with $\mathcal{T}$ torsion and $\mathcal{E}_{0}$ and $\mathcal{E}_{1}$ locally free, and $\mathcal{F}$ a coherent torsion-free sheaf on $S$. Then

(1) $H^{0}\left(S, \mathcal{E} x t^{1}(\mathcal{T}, \mathcal{F})\right)=\operatorname{Ext}^{1}(\mathcal{T}, \mathcal{F})$;

(2) for any morphism $f: R \rightarrow S, f^{*} \mathcal{E} x t_{S}^{1}(\mathcal{T}, \mathcal{F})=\mathcal{E} x t_{R}^{1}\left(f^{*} \mathcal{T}, f^{*} \mathcal{F}\right)$.

Proof. (1) Since $\mathcal{T}$ is torsion and $\mathcal{F}$ is torsion-free, we have $\operatorname{Hom}(\mathcal{T}, \mathcal{F})=0$ and $\mathcal{H o m}(\mathcal{T}, \mathcal{F})=0$. Applying $\operatorname{Hom}(-, \mathcal{F})$ and $\mathcal{H o m}(-, \mathcal{F})$ to the locally free presentation of $\mathcal{T}$, we obtain a long exact sequence

$$
0 \rightarrow \operatorname{Hom}\left(\mathcal{E}_{0}, \mathcal{F}\right) \rightarrow \operatorname{Hom}\left(\mathcal{E}_{1}, \mathcal{F}\right) \rightarrow \operatorname{Ext}^{1}(\mathcal{T}, \mathcal{F}) \rightarrow \operatorname{Ext}^{1}\left(\mathcal{E}_{0}, \mathcal{F}\right) \rightarrow \operatorname{Ext}^{1}\left(\mathcal{E}_{1}, \mathcal{F}\right)
$$

and a short exact sequence

$$
0 \rightarrow \mathcal{H o m}\left(\mathcal{E}_{0}, \mathcal{F}\right) \rightarrow \mathcal{H o m}\left(\mathcal{E}_{1}, \mathcal{F}\right) \rightarrow \mathcal{E} x t^{1}(\mathcal{T}, \mathcal{F}) \rightarrow 0
$$

Taking global sections of the above short exact sequence, we obtain a long exact sequence:

$$
\begin{aligned}
0 \rightarrow H^{0}\left(\mathcal{H o m}\left(\mathcal{E}_{0}, \mathcal{F}\right)\right) \rightarrow H^{0}\left(\mathcal{H o m}\left(\mathcal{E}_{1}, \mathcal{F}\right)\right) \rightarrow H^{0}\left(\mathcal{E} x t^{1}(\mathcal{T}, \mathcal{F})\right) \\
\rightarrow H^{1}\left(\mathcal{H o m}\left(\mathcal{E}_{0}, \mathcal{F}\right)\right) \rightarrow H^{1}\left(\mathcal{H o m}\left(\mathcal{E}_{1}, \mathcal{F}\right)\right)
\end{aligned}
$$

We have identifications

$$
\operatorname{Hom}\left(\mathcal{E}_{i}, \mathcal{F}\right)=H^{0}\left(\mathcal{H o m}\left(\mathcal{E}_{i}, \mathcal{F}\right)\right), \quad i=0,1
$$


and a natural homomorphism

$$
\operatorname{Ext}^{1}(\mathcal{T}, \mathcal{F}) \rightarrow H^{0}\left(\mathcal{E} x t^{1}(\mathcal{T}, \mathcal{F})\right)
$$

Using the Grothendieck spectral sequence for the composition of the two functors $H^{0}$ and $\mathcal{H o m}^{1}\left(\mathcal{E}_{i},-\right)$, we obtain exact sequences of low degrees:

$$
0 \rightarrow H^{1}\left(\mathcal{H o m}\left(\mathcal{E}_{i}, \mathcal{F}\right)\right) \rightarrow \operatorname{Ext}^{1}\left(\mathcal{E}_{i}, \mathcal{F}\right) \rightarrow H^{0}\left(\mathcal{E} x t^{1}\left(\mathcal{E}_{i}, \mathcal{F}\right)\right) \rightarrow \cdots, \quad i=0,1 .
$$

Since $\mathcal{E}_{i}$ are locally free, we have $H^{0}\left(\mathcal{E} x t^{1}\left(\mathcal{E}_{i}, \mathcal{F}\right)\right)=0$. Therefore we obtain identifications:

$$
H^{1}\left(\mathcal{H o m}\left(\mathcal{E}_{i}, \mathcal{F}\right)\right)=\operatorname{Ext}^{1}\left(\mathcal{E}_{i}, \mathcal{F}\right), \quad i=0,1
$$

Thus we have a commutative diagram

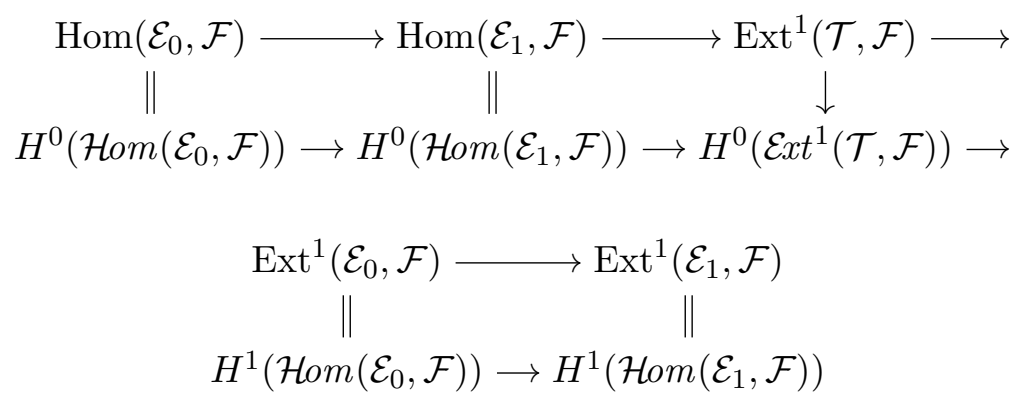

By the Five Lemma, we obtain the identification

$$
H^{0}\left(\mathcal{E} x t^{1}(\mathcal{T}, \mathcal{F})\right)=\operatorname{Ext}^{1}(\mathcal{T}, \mathcal{F})
$$

The proof of (2) is parallel to that of [Shao11], Proposition 2.3. So, we omit the details.

2.2. The space of relative non-split extensions. To proceed, we fix a set of notations.

Notation. For any $\mathbb{k}$-schemes $R$ and $S$,

(1) we denote the projection $\mathbb{P}^{1} \times R \rightarrow R$ by $\pi_{R}$ or simply $\pi$;

(2) for any coherent sheaf $\mathcal{H}$ over $R$, denote by $\mathcal{H}^{\vee}$ the dual sheaf $\mathcal{H o m}_{R}\left(\mathcal{H}, \mathcal{O}_{R}\right)$;

(3) for any coherent sheaf $\mathcal{F}$ on $\mathbb{P}^{1} \times R$, we set $\mathcal{F}_{x}:=\left.\mathcal{F}\right|_{\mathbb{P}^{1} \times\{x\}}$ for any point $x \in R$;

(4) for any morphism $f: R \rightarrow S$, we set $\bar{f}:=1 \times f: \mathbb{P}^{1} \times R \rightarrow \mathbb{P}^{1} \times S$. 
We now introduce a relative version of the space of non-split extensions. Let $S$ be a noetherian scheme over $\mathbb{k}$ and let $\mathcal{F}$ and $\mathcal{T}$ be two coherent sheaves on $\mathbb{P}^{1} \times S$, both flat over $S$, with $\mathcal{F}$ locally free and $\mathcal{T}$ torsion (i.e., having rank 0 ). Let $\pi_{S}: \mathbb{P}^{1} \times S \rightarrow S$ be the projection, and set

$$
\mathcal{E}:=\pi_{S *} \mathcal{E}^{1} t_{\mathbb{P}^{1} \times S}^{1}(\mathcal{T}, \mathcal{F}) .
$$

As in [Shao11], Proposition $2.4(1)$, one checks that $\mathcal{E} x t^{1}(\mathcal{T}, \mathcal{F})$ is a torsion sheaf and is flat over $S$. By Cohomology and Base Change, we can show $\mathcal{E}$ is locally free and for any point $s \in S$,

$$
\left.\mathcal{E}\right|_{s}=H^{0}\left(\mathcal{E} x t^{1}(\mathcal{T}, \mathcal{F})_{s}\right)=H^{0}\left(\mathcal{E} x t^{1}\left(\mathcal{T}_{s}, \mathcal{F}_{s}\right)\right)=\operatorname{Ext}^{1}\left(\mathcal{T}_{s}, \mathcal{F}_{s}\right)
$$

where the second equality holds by Lemma 2.1 (2) and the third holds by Lemma 2.1 (1). So the projective bundle

$$
\mathbb{P}(\mathcal{E}):=\operatorname{Proj}\left(\operatorname{Sym}^{*}\left(\mathcal{E}^{\vee}\right)\right)
$$

over $S$ is a family of spaces of non-split extensions: for each point $s \in S$, the fiber of $\mathbb{P}(\mathcal{E})$ over $s$ is $\mathbb{P}\left(\left.\mathcal{E}\right|_{s}\right)=\mathbb{P}\left(\operatorname{Ext}^{1}\left(\mathcal{T}_{s}, \mathcal{F}_{s}\right)\right)$.

\subsection{The universal extension.}

Notation. For any coherent sheaf $H$ on $\mathbb{P}^{1} \times \mathbb{P}(\mathcal{E})$, we denote by $H(m, n)$ the sheaf

$$
H \otimes p^{*} \mathcal{O}_{\mathbb{P}^{1}}(m) \otimes \pi_{\mathbb{P}(\mathcal{E})}^{*} \mathcal{O}_{\mathbb{P}(\mathcal{E})}(n)
$$

where $p: \mathbb{P}^{1} \times \mathbb{P}(\mathcal{E}) \rightarrow \mathbb{P}^{1}$ and $\pi_{\mathbb{P}(\mathcal{E})}: \mathbb{P}^{1} \times \mathbb{P}(\mathcal{E}) \rightarrow \mathbb{P}(\mathcal{E})$ are the two projections.

Let $a: \mathbb{P}(\mathcal{E}) \rightarrow S$ be the structure morphism. The space $\mathbb{P}(\mathcal{E})$ comes equipped with a universal quotient $a^{*} \mathcal{E}^{\vee} \rightarrow \mathcal{O}_{\mathbb{P}(\mathcal{E})}(1)$. Dualizing the universal quotient and tensoring the result with $\mathcal{O}_{\mathbb{P}(\mathcal{E})}(1)$, we obtain a line subbundle

$$
\begin{gathered}
\mathcal{O}_{\mathbb{P}(\mathcal{E})} \hookrightarrow a^{*} \mathcal{E} \otimes \mathcal{O}_{\mathbb{P}(\mathcal{E})}(1)=\pi_{\mathbb{P}(\mathcal{E}) *} \bar{a}^{*} \mathcal{E} x t^{1}(\mathcal{T}, \mathcal{F}) \otimes \mathcal{O}_{\mathbb{P}(\mathcal{E})}(1)= \\
\pi_{\mathbb{P}(\mathcal{E}) *}\left(\mathcal{E} x t^{1}\left(\bar{a}^{*} \mathcal{T}, \bar{a}^{*} \mathcal{F}\right)(0,1)\right)=\pi_{\mathbb{P}(\mathcal{E}) *} \mathcal{E} x t^{1}\left(\bar{a}^{*} \mathcal{T}, \bar{a}^{*} \mathcal{F}(0,1)\right)
\end{gathered}
$$

Here the first equality holds because the morphism $a$ is flat, and the second holds by Lemma 2.1 (2). This line subbundle corresponds to a nonzero element of

$$
\begin{aligned}
\Gamma\left(\mathbb{P}(\mathcal{E}), \pi_{\mathbb{P}(\mathcal{E}) *} \mathcal{E} x t^{1}\left(\bar{a}^{*} \mathcal{T}, \bar{a}^{*} \mathcal{F}(0,1)\right)\right) & =\Gamma\left(\mathbb{P}^{1} \times \mathbb{P}(\mathcal{E}), \mathcal{E} x t^{1}\left(\bar{a}^{*} \mathcal{T}, \bar{a}^{*} \mathcal{F}(0,1)\right)\right) \\
& =\operatorname{Ext}^{1}\left(\bar{a}^{*} \mathcal{T}, \bar{a}^{*} \mathcal{F}(0,1)\right)
\end{aligned}
$$


where the second equality holds by Lemma 2.1 (1). We can write this element as an extension

$$
0 \rightarrow \bar{a}^{*} \mathcal{F}(0,1) \rightarrow \mathcal{X} \rightarrow \bar{a}^{*} \mathcal{T} \rightarrow 0 .
$$

which we call the universal extension. Note that the universal extension is nowhere-split, which means that, for each $\mathbb{k}$-point $s \in S$ and each $\mathbb{k}$-point $x \in$ $a^{-1}(\{s\})=\mathbb{P}\left(\operatorname{Ext}^{1}\left(\mathcal{T}_{s}, \mathcal{F}_{s}\right)\right)$, the extension

$$
0 \rightarrow \mathcal{F}_{s} \rightarrow \mathcal{X}_{x} \rightarrow \mathcal{T}_{s} \rightarrow 0
$$

obtained by pulling back the universal extension to $\mathbb{P}^{1} \times\{x\} \simeq \mathbb{P}^{1}$ is non-split.

Conversely, the universal quotient can be recovered from the universal extension by reversing the above process. In fact, the universal extension itself is a nonzero element of

$$
\begin{aligned}
& \operatorname{Ext}^{1}\left(\bar{a}^{*} \mathcal{T}, \bar{a}^{*} \mathcal{F}(0,1)\right)=\Gamma\left(\mathbb{P}^{1} \times \mathbb{P}(\mathcal{E}), \mathcal{E} x t^{1}\left(\bar{a}^{*} \mathcal{T}, \bar{a}^{*} \mathcal{F}(0,1)\right)\right. \\
& =\Gamma\left(\mathbb{P}(\mathcal{E}), \pi_{\mathbb{P}(\mathcal{E}) *} \mathcal{E} x t^{1}\left(\bar{a}^{*} \mathcal{T}, \bar{a}^{*} \mathcal{F}(0,1)\right)\right)=\Gamma\left(\mathbb{P}(\mathcal{E}), a^{*} \mathcal{E}(1)\right)
\end{aligned}
$$

hence determines a line subbundle $\mathcal{O}_{\mathbb{P}(\mathcal{E})} \hookrightarrow a^{*} \mathcal{E}(1)$. Tensoring the line subbundle with $\mathcal{O}_{\mathbb{P}(\mathcal{E})}(-1)$ and taking the dual, we obtain the universal quotient.

Thus, based on the universal property of the universal quotient, we have

Theorem 2.2. Let $R$ be an $S$-scheme with structure morphism $\rho: R \rightarrow S, L$ a line bundle on $R$. If $0 \rightarrow \bar{\rho}^{*} \mathcal{F} \otimes \pi_{R}^{*} L \rightarrow \mathcal{Y} \rightarrow \bar{\rho}^{*} \mathcal{T} \rightarrow 0$ is a nowhere-split extension on $\mathbb{P}^{1} \times R$, then there is a unique $S$-morphism $f: R \rightarrow \mathbb{P}(\mathcal{E})$ such that there are isomorphisms $L \stackrel{g}{\simeq} f^{*} \mathcal{O}_{\mathbb{P}(\mathcal{E})}(1), \mathcal{Y} \stackrel{h}{\simeq} \bar{f}^{*} \mathcal{X}$ that make the following diagram commute:

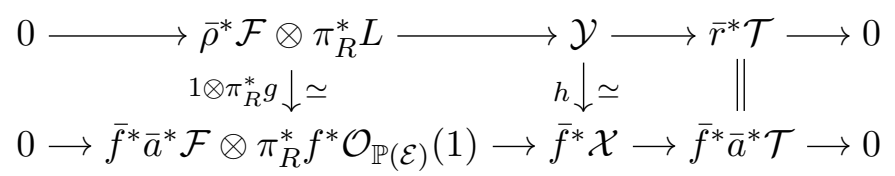

where the second row is the pullback of the universal extension via $\bar{f}$.

\section{A fLATTENing STRATificAtion}

In this section, we continue to follow the previous notations, and we impose an additional assumption on the sheaf $\mathcal{F}$ of Theorem 2.2 (also Lemma 2.1):

$$
R^{1} \pi_{S *}\left(\mathcal{F} \otimes p^{*} \mathcal{O}_{\mathbb{P}^{1}}(-1)\right)=0,
$$


where $p: \mathbb{P}^{1} \times S \rightarrow \mathbb{P}^{1}$ is the projection.

We also need the following notation (cf. Introduction).

Notation. For any coherent sheaf $H$ on $\mathbb{P}^{1}$, we denote by $H^{\mathbf{t}}$ the torsion subsheaf of $H$. The quotient $H / H^{\mathbf{t}}$ is locally free and we will denote it by $H^{\mathbf{f}}$. Since $H \simeq H^{\mathbf{t}} \oplus H^{\mathbf{f}}$ on $\mathbb{P}^{1}$, we call $H^{\mathbf{t}}$ and $H^{\mathbf{f}}$ the torsion part and the locally free part of $H$, respectively.

Let $d_{1}$ and $d_{2}$ be the relative degrees of $\mathcal{F}$ and $\mathcal{T}$ over $S$, respectively. Then the universal extention $\mathcal{X}$ (see (2.1)) is of relative degree $d:=d_{1}+d_{2}$ over $S$. The set

$$
\stackrel{\circ}{\mathbb{P}}(\mathcal{E}):=\left\{x \in \mathbb{P}(\mathcal{E}) \mid \mathcal{X}_{x} \text { is locally free }\right\}
$$

is an open subset of $\mathbb{P}(\mathcal{E})$. Its complement $\mathbb{P}(\mathcal{E}) \backslash \stackrel{\circ}{P}(\mathcal{E})$ has a sequence of nested closed subsets:

$$
\varnothing=Y_{d_{1}} \subset Y_{d_{1}+1} \subset \cdots \subset Y_{d-1}=\mathbb{P}(\mathcal{E}) \backslash \stackrel{\circ}{\mathbb{P}}(\mathcal{E})
$$

where $d$ is the relative degree of $\mathcal{T}$ over $S$, and

$$
Y_{r}=\left\{x \in \mathbb{P}(\mathcal{E}) \mid \operatorname{deg}\left(\left(\mathcal{X}_{x}\right)^{\mathbf{t}}\right) \geq d-r\right\}, \quad r=d_{1}, \ldots, d-1 .
$$

Note that an extension $0 \rightarrow \mathcal{F}_{s} \rightarrow X \rightarrow \mathcal{T}_{s} \rightarrow 0$ is split if and only if the torsion part of $X$ is of degree $d_{2}$. Hence $Y_{d_{1}}=\varnothing$.

For $m \geq 0$, applying $\operatorname{Hom}(-, \mathcal{O}(m))$ to the extension $(2.2)$, we obtain an exact sequence

$0 \rightarrow \operatorname{Hom}\left(\mathcal{X}_{x}, \mathcal{O}(m)\right) \rightarrow \operatorname{Hom}\left(\mathcal{F}_{s}, \mathcal{O}(m)\right) \stackrel{\delta_{x, m}}{\rightarrow} \operatorname{Ext}^{1}\left(\mathcal{T}_{s}, \mathcal{O}(m)\right) \rightarrow \operatorname{Ext}^{1}\left(\mathcal{X}_{x}, \mathcal{O}(m)\right) \rightarrow 0$

where $\delta_{x, m}$ denotes the connecting homomorphism. Then we have

Proposition 3.1. $\operatorname{rank} \delta_{x, m}=d_{2}-\operatorname{deg}\left(\mathcal{X}_{x}\right)^{\mathbf{t}}$ for any $m \geq d$.

Proof. Suppose $\mathcal{F}_{s}$ has rank $k$. Then $\mathcal{X}_{x}$ has rank $k$ as well. We have noncanonical isomorphisms

$$
\mathcal{F}_{s} \simeq \bigoplus_{i=1}^{r} \mathcal{O}\left(a_{i}\right), \quad \mathcal{X}_{x} \simeq\left(\mathcal{X}_{x}\right)^{\mathbf{t}} \oplus \bigoplus_{i=1}^{r} \mathcal{O}\left(b_{i}\right)
$$

where $\sum_{i=1}^{r} a_{i}=d_{1}$ and $\sum_{i=1}^{r} b_{i}=d-\operatorname{deg}\left(\mathcal{X}_{x}\right)^{\mathbf{t}}$. By the assumption (3.1), we have $H^{1}\left(\mathbb{P}^{1}, \mathcal{F}_{s}(-1)\right)=0$ and also $H^{1}\left(\mathbb{P}^{1}, \mathcal{X}_{x}(-1)\right)=0$. It follows that $a_{i} \geq 0$ 
and $b_{i} \geq 0$ for all $i$. Hence we have $a_{i} \leq d_{1}<d$ and $b_{i} \leq d$ for all $i$. So

$$
\begin{gathered}
\operatorname{Hom}\left(\mathcal{F}_{s}, \mathcal{O}(m)\right) \simeq \bigoplus_{i=1}^{k} \operatorname{Hom}\left(\mathcal{O}\left(a_{i}\right), \mathcal{O}(m)\right)=\bigoplus_{i=1}^{k} H^{0}\left(\mathcal{O}\left(m-a_{i}\right)\right), \text { and } \\
\operatorname{Hom}\left(\mathcal{X}_{x}, \mathcal{O}(m)\right) \simeq \bigoplus_{i=1}^{k} \operatorname{Hom}\left(\mathcal{O}\left(b_{i}\right), \mathcal{O}(m)\right)=\bigoplus_{i=1}^{k} H^{0}\left(\mathcal{O}\left(m-b_{i}\right)\right)
\end{gathered}
$$

For $m \geq d$, we have $a_{i} \leq m$ and $b_{i} \leq m$, therefore

$$
\begin{aligned}
\operatorname{rank} \delta_{x, m} & =\operatorname{dim} \operatorname{Hom}\left(\mathcal{F}_{s}, \mathcal{O}(m)\right)-\operatorname{dim} \operatorname{Hom}\left(\mathcal{X}_{x}, \mathcal{O}(m)\right) \\
& =\sum_{i=1}^{k}\left(m-a_{i}+1\right)-\left(m-b_{i}+1\right) \\
& =\sum_{i=1}^{k} b_{i}-\sum_{i=1}^{k} a_{i}=d-\operatorname{deg}\left(\mathcal{X}_{x}\right)^{\mathbf{t}}-d_{1}=d_{2}-\operatorname{deg}\left(\mathcal{X}_{x}\right)^{\mathbf{t}}
\end{aligned}
$$

Corollary 3.2. For any integers $m, r$ with $m \geq d$ and $d_{1} \leq r \leq d$, we have $\operatorname{deg}\left(\mathcal{X}_{x}\right)^{\mathbf{t}} \geq d-r$ if and only if $\operatorname{rank} \delta_{x, m} \leq r-d_{1}$.

Notation. For any scheme $R$ and any coherent sheaf $\mathcal{H}$ on $R$, we will use the following abbreviations:

$$
\mathcal{H}^{\varepsilon}:=\mathcal{E} x t_{R}^{1}\left(\mathcal{H}, \mathcal{O}_{R}\right)
$$

Corollary 3.2 suggests us that we can define the scheme structure of $Y_{r}$ in the following way. Let $m \gg 0$. Applying $\mathcal{H o m}\left(-, \mathcal{O}_{\mathbb{P} 1} \times \mathbb{P}(\mathcal{E})(m, 1)\right)$ to the universal extension (2.1) to obtain an exact sequence

$$
0 \rightarrow \mathcal{X}^{\vee}(m, 1) \rightarrow \bar{a}^{*} \mathcal{F}^{\vee}(m, 0) \stackrel{\delta_{m}}{\rightarrow} \bar{a}^{*} \mathcal{T}^{\varepsilon}(m, 1) \rightarrow \mathcal{X}^{\varepsilon}(m, 1) \rightarrow 0
$$

where $\delta_{m}$ is the connecting homomorphism. Next, applying $\pi_{\mathbb{P}(\mathcal{E}) *}$ to $\delta_{m}$ and using identifications

$$
\begin{aligned}
& \pi_{*}\left(\bar{a}^{*} \mathcal{F}^{\vee}(m, 0)\right)=\pi_{*} \bar{a}^{*}\left(\mathcal{F}^{\vee}(m)\right)=a^{*}\left(\pi_{*} \mathcal{F}^{\vee}(m)\right), \\
& \pi_{*}\left(\bar{a}^{*} \mathcal{T}^{\varepsilon}(m, 1)\right)=\pi_{*} \bar{a}^{*}\left(\mathcal{T}^{\varepsilon}(m)\right)(1)=a^{*}\left(\pi_{*} \mathcal{T}^{\varepsilon}(m)\right)(1)
\end{aligned}
$$

we obtain a nowhere-vanishing homomorphism

$$
\pi_{*} \delta_{m}: a^{*}\left(\pi_{*} \mathcal{F}^{\vee}(m)\right) \rightarrow a^{*}\left(\pi_{*} \mathcal{T}^{\varepsilon}(m)\right)(1)
$$


Note that both $\pi_{*} \mathcal{F}^{\vee}(m)$ and $\pi_{*} \mathcal{T}^{\varepsilon}(m)$ are locally free sheaves on $S$ for $m \gg 0$. Applying the exterior power $\bigwedge^{l+1}(l \geq 0)$ to $\pi_{*} \delta_{m}$, we obtain

$$
\bigwedge^{l+1} \pi_{*} \delta_{m}: \bigwedge^{l+1} a^{*}\left(\pi_{*} \mathcal{F}^{\vee}(m)\right) \rightarrow \bigwedge^{l+1} a^{*}\left(\pi_{*} \mathcal{T}^{\varepsilon}(m)\right)(l+1)
$$

By Corollary 3.2, $Y_{d_{1}+l}$ is exactly the (set-theoretic) zero locus of $\bigwedge^{l+1} \pi_{*} \delta_{m}$, for each $l$ with $0 \leq l<d_{2}$. The section $\bigwedge^{l+1} \pi_{*} \delta_{m}$ induces a homomorphism

$$
\mathcal{H o m}\left(\bigwedge^{l+1} a^{*}\left(\pi_{*} \mathcal{F}^{\vee}(m)\right), \bigwedge^{l+1} a^{*}\left(\pi_{*} \mathcal{T}^{\varepsilon}(m)\right)(l+1)\right)^{\vee} \rightarrow \mathcal{O}_{\mathbb{P}(\mathcal{E})}
$$

The image, which we denote by $I_{l, m}$, is an ideal sheaf. In a similar way as in [Shao11], Proposition 3.4, we can prove that

Proposition 3.3. There exists an integer $N>0$ such that $I_{l, m}=I_{l, N}$ as subsheaves of $\mathcal{O}_{\mathbb{P}(\mathcal{E})}$ for all $m>N$ and for all $l$ with $0 \leq l<d_{2}$.

Obviously, the subscheme defined by the ideal $I_{l, N}$ is supported on the closed subset $Y_{d_{1}+l}$. For simplicity, we denote this subscheme still by $Y_{d_{1}+l}$, that is,

$Y_{r}=$ the closed subscheme defined by the ideal $I_{r-d_{1}+1, N}, \quad r=d_{1}+1, \ldots, d-1$

In addition, we set $\stackrel{\circ}{Y}_{r}:=Y_{r} \backslash Y_{r-1}$ for $d_{1}<r<d$. Note that ${\stackrel{\circ}{d_{1}+1}}_{Y_{d}}=Y_{d_{1}+1}$ since $Y_{d_{1}}=\varnothing$.

Again, in a similar way as in [Shao11], Theorem 3.5, we can prove that

Proposition 3.4. The locally closed subschemes $Y_{d_{1}+1},{\stackrel{\circ}{Y_{d}+2}}_{d_{1}}, \cdots, \stackrel{\circ}{Y}_{d-1}$ of $\mathbb{P}(\mathcal{E})$ form the flattening stratification of $\mathbb{P}(\mathcal{E})$ by the sheaf $\mathcal{X}^{\varepsilon}$, which means that, for any noetherian $\mathbb{k}$-scheme $R$ and any morphism $f: R \rightarrow \mathbb{P}(\mathcal{E})$, the sheaf $\bar{f}^{*} \mathcal{X}^{\varepsilon}$ on $\mathbb{P}^{1} \times R$ is flat over $R$ with relative degree $d-r$ if and only if $f$ factors through the inclusion $\stackrel{\circ}{Y}_{r} \hookrightarrow \mathbb{P}(\mathcal{E})$. In particular, the restriction of $\mathcal{X}^{\varepsilon}$ to $\mathbb{P}^{1} \times \stackrel{\circ}{Y}_{r}$ is flat over $\stackrel{\circ}{Y}_{r}$ with relative degree $d-r$.

\section{The nORMal Bundles: A FIRST CASE}

Let $V$ be a vector space of dimension $n$ over $\mathbb{k}$, and $\operatorname{Gr}(k, V)$ be the Grassmannian parametrizing all the $k$-dimensional subspaces of $V$. For any $d \geq 0$, the space $\operatorname{Mor}_{d}\left(\mathbb{P}^{1}, \operatorname{Gr}(k, V)\right)$ of degree $d$ maps from $\mathbb{P}^{1}$ to $\operatorname{Gr}(k, V)$ is a nonsingular quasi-projective variety. A smooth compactification of $\operatorname{Mor}_{d}\left(\mathbb{P}^{1}, \operatorname{Gr}(k, V)\right)$ is 
given by the Quot scheme $Q_{d}:=\operatorname{Quot}_{V_{\mathbb{P} 1} / \mathbb{P}^{1} / \mathbb{k}}^{n-k, d}$, parametrizing all rank- $(n-k)$, degree- $d$ quotients $V_{\mathbb{P}^{1}} \rightarrow F$ of the trivial vector bundle $V_{\mathbb{P}^{1}}$ of rank $n$ on $\mathbb{P}^{1}$. It comes with a universal exact sequence of sheaves on $\mathbb{P}^{1} \times Q_{d}$ :

$$
0 \rightarrow \mathcal{E}_{d} \rightarrow V_{\mathbb{P}^{1} \times Q_{d}} \rightarrow \mathcal{F}_{d} \rightarrow 0
$$

Here $\mathcal{F}_{d}$ is flat over $Q_{d}$ with rank $n-k$ and relative degree $d$. It follows that $\mathcal{E}_{d}$ is locally free with rank $k$ and relative degree $-d$ over $Q_{d}$. The open subvariety $\stackrel{\circ}{d}_{d}:=\left\{x \in Q_{d} \mid\left(\mathcal{F}_{d}\right)_{x}\right.$ is locally free $\}$ coincides with $\operatorname{Mor}_{d}\left(\mathbb{P}^{1}, \operatorname{Gr}(k, V)\right)$.

Recall from the introduction that for any $d>r \geq 0$, we have the closed subscheme

$$
Z_{d, r}=\left\{\left[V_{\mathbb{P}^{1}} \rightarrow F\right] \in Q_{d} \mid \operatorname{deg}\left(F^{\mathbf{t}}\right) \geq d-r\right\} .
$$

We refer the reader to Section 3 in [Shao11] for the details on the subscheme structure of $Z_{d, r}$. These are the subschemes that are blown up to yield the variety $\widetilde{Q}_{d}$. Below we analyze the normal bundle of the locally closed subsets $\stackrel{\circ}{d, r}_{\text {in }}:=Z_{d, r} \backslash Z_{d, r-1}$.

The subscheme $Z_{d, r}$ is closely related to the following relative Quot scheme over $Q_{r}$ :

$$
Q_{d, r}:=\operatorname{Quot}_{\mathcal{E}_{r} / \mathbb{P}^{1} \times Q_{r} / Q_{r}}^{0, d-r}
$$

If a point of $Q_{r}$ is represented by the exact sequence $E \longmapsto V_{\mathbb{P} 1} \rightarrow F$, then the fiber of $Q_{d, r}$ over the point consists of points represented by the quotient $E \rightarrow T$ with $T$ torsion of degree $d-r$. Let $\theta_{d, r}: Q_{d, r} \rightarrow Q_{r}$ be the structure morphism. (Note that the notation for this morphism is simply $\theta$ in [Shao11]. We add sub-index in this paper because we will deal with multiple $Q_{d, r}$ 's together with their structure morphisms simultaneously. The same reason applies to the other similar situations below.) In [Shao11], we showed that $Q_{d, r}$ is relatively smooth over $Q_{r}$, hence is a nonsingular variety. It comes equipped with a universal exact sequence on $\mathbb{P}^{1} \times Q_{d, r}$ :

$$
0 \rightarrow \mathcal{E}_{d, r} \rightarrow \theta_{d, r}^{*} \mathcal{E}_{r} \rightarrow \mathcal{T}_{d, r} \rightarrow 0
$$

Here $\mathcal{T}_{d, r}$ is flat over $Q_{d, r}$ with rank 0 and relative degree $d-r$. Since $\theta_{d, r}^{*} \mathcal{E}_{r}$ is locally free of rank $k$ and of relative degree $-r$ over $Q_{d, r}$, it follows that $\mathcal{E}_{d, r}$ is locally free of rank $k$ and of relative degree $-d$ over $Q_{d, r}$. We set $\stackrel{\circ}{d, r}_{d}:=\theta_{d, r}^{-1}\left(\stackrel{\circ}{Q}_{r}\right)$ and write $\stackrel{\circ}{\theta}_{d, r}: \stackrel{\circ}{Q}_{d, r} \rightarrow Q_{r}$ for the restriction of $\theta_{d, r}$ on $\stackrel{\circ}{d, r}_{d}$. 
We form a commutative diagram on $\mathbb{P}^{1} \times Q_{d, r}$ :

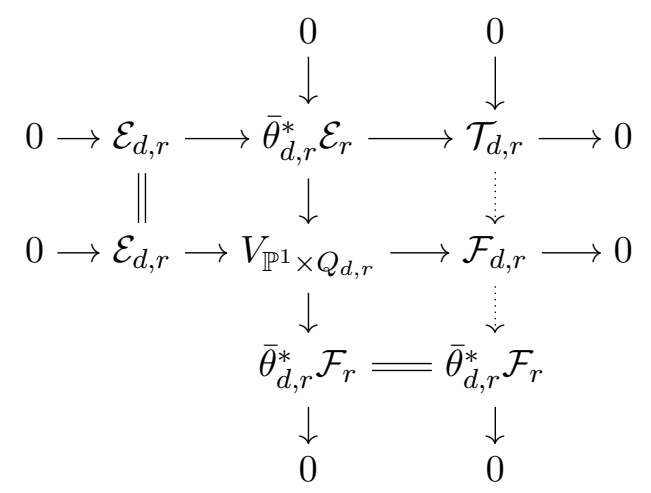

where the middle column is the pullback of the universal exact sequence of $Q_{r}$ via $\bar{\theta}_{d, r}$, and $\mathcal{F}_{d, r}$ is defined to be the cokernel of the composite map $\mathcal{E}_{d, r} \rightarrow \bar{\theta}_{d, r}^{*} \mathcal{E}_{r} \rightarrow$ $V_{\mathbb{P} 1} \times Q_{d, r}$. One checks that $\mathcal{F}_{d, r}$ is flat over $Q_{d, r}$ of rank $n-k$ and relative degree $d$. By the universal property of $Q_{d}$, the middle row determines a morphism $\phi_{d, r}: Q_{d, r} \rightarrow Q_{d}$ (it is denoted by $\phi$ in [Shao11]) such that the following diagram commutes:

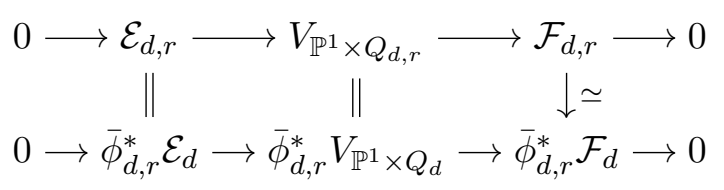

The morphism $\phi_{d, r}$ maps $Q_{d, r}$ onto $Z_{d, r}$ (cf. Proposition 4.6 of [Shao11]). We

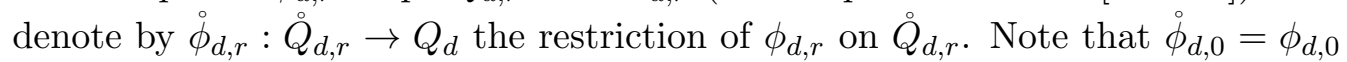

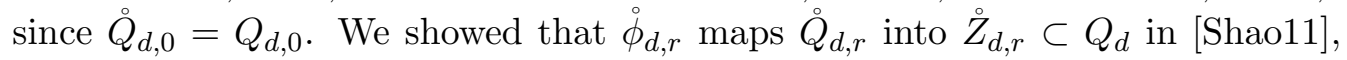
where $\stackrel{\circ}{Z}_{d, r}=Z_{d, r} \backslash Z_{d, r-1}$, and we set $Z_{d,-1}:=\varnothing$. We denote by $\stackrel{\circ}{\varphi, r}_{d,}: \stackrel{\circ}{Q}_{d, r} \rightarrow \stackrel{\circ}{Z}_{d, r}$ the map obtained by restricting the codomain of $\stackrel{\circ}{\phi}_{d, r}$ to $\stackrel{\circ}{Z}_{d, r}$. In [Shao11], we

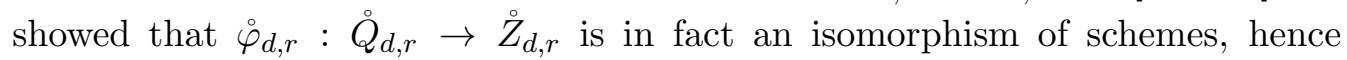
$\stackrel{\circ}{\phi}, r_{Q_{d, r}} \rightarrow Q_{d}$ is an embedding. (cf. Proposition 4.8, [Shao11])

Proposition 4.1 ([Str87], Theorem 7.1). The tangent bundle $\mathcal{T}_{Q_{d}}$ of $Q_{d}$ is naturally isomorphic to $\pi_{*} \mathcal{H}$ om $\left(\mathcal{E}_{d}, \mathcal{F}_{d}\right)$. The relative tangent bundle $\mathcal{T}_{Q_{d, r}} / Q_{r}$ of $Q_{d, r}$ over $Q_{r}$ is naturally isomorphic to $\pi_{*} \mathcal{H o m}\left(\mathcal{E}_{d, r}, \mathcal{T}_{d, r}\right)$.

Proof. The first assertion is proved in [Str87], Theorem 7.1. The second, which is a relative version of the first, can be proved by slightly modifying the proof of [Str87], Theorem 7.1. We omit the details. 
The tangent bundle $\mathcal{T}_{Q_{d, r}}$ of $Q_{d, r}$ fits into the following exact sequence

$$
0 \rightarrow \mathcal{T}_{Q_{d, r} / Q_{r}} \rightarrow \mathcal{T}_{Q_{d, r}} \rightarrow \theta_{d, r}^{*} \mathcal{T}_{Q_{r}} \rightarrow 0
$$

The morphism $\dot{\phi}_{d, r}: \stackrel{\circ}{d, r}_{R} \rightarrow Q_{d}$ is an embedding and is factored as

$$
\stackrel{\circ}{Q}_{d, 0} \stackrel{\stackrel{\circ}{\varphi}_{d, r}}{\rightarrow} \stackrel{\circ}{Z}_{d, r} \hookrightarrow Q_{d}
$$

(cf. Proposition 4.8 of [Shao11]). Let $\mathcal{N}_{\stackrel{Q}{d, r} / Q_{d}}$ denote the normal sheaf of $\AA_{d, 0}$ in $Q_{d}$. Since $Q_{d, r}$ is nonsingular, $\mathcal{N}_{\AA_{d, r} / Q_{d}}$ is locally free.

Proposition 4.2. We have a natural identification

$$
\mathcal{N}_{\AA_{d, r} / Q_{d}}=\left.\pi_{*} \mathcal{E} x t^{1}\left(\mathcal{T}_{d, r}, \bar{\theta}_{d, r}^{*} \mathcal{F}_{r}\right)\right|_{\AA_{d, r}} .
$$

In particular, letting $r=0$, we have $\mathcal{N}_{Q_{d, 0} / Q_{d}}=\pi_{*} \mathcal{E} x t^{1}\left(\mathcal{T}_{d, 0}, \bar{\theta}_{d, 0}^{*} \mathcal{F}_{0}\right)$.

Proof. Since $\mathcal{N}_{\AA_{d, r} / Q_{d}}$ is locally free, we have the following exact sequence of sheaves on $\stackrel{\circ}{d, r}_{d}$ :

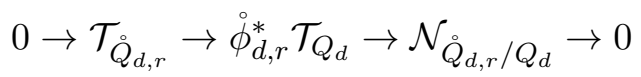

Note that the restriction of the exact sequence (4.4) to $\stackrel{\circ}{Q}_{d, r}$ gives an exact sequence

$$
0 \rightarrow \mathcal{T}_{\stackrel{\circ}{Q}_{d, r} / \stackrel{\circ}{Q}_{r}} \rightarrow \mathcal{T}_{\stackrel{\circ}{Q}_{d, r}} \rightarrow \stackrel{\circ}{\theta}_{d, r}^{*} \mathcal{T}_{Q_{r}} \rightarrow 0
$$

Combining the above two sequences, we can form a commutative diagram of sheaves on $\stackrel{\circ}{Q}_{d, r}$ :

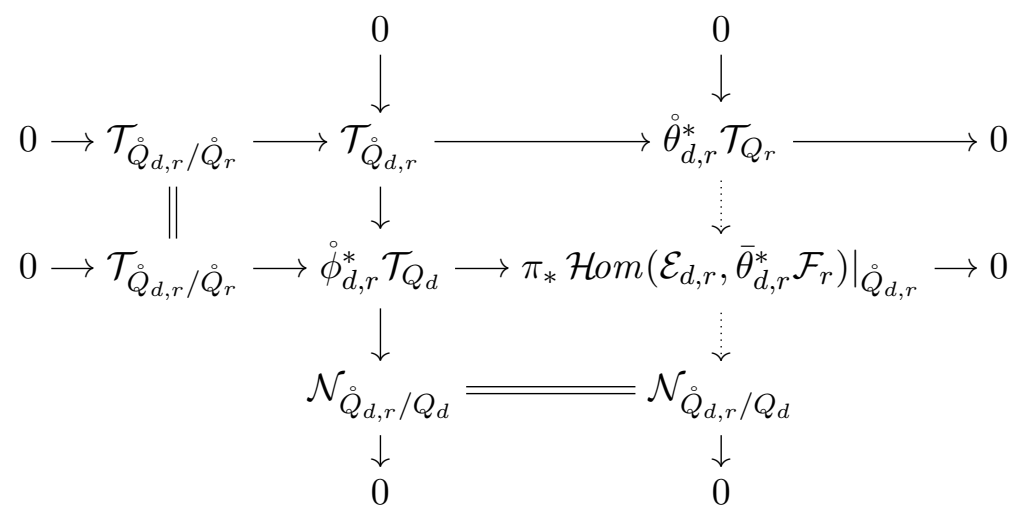

where the middle row comes from the natural identifications

$$
\begin{aligned}
& \mathcal{T}_{\grave{Q}_{d, r} / \AA^{\circ}}=\left.\pi_{*} \mathcal{H o m}\left(\mathcal{E}_{d, r}, \mathcal{T}_{d, r}\right)\right|_{\AA_{d, r}}, \\
& \stackrel{\circ}{d, r}^{*} \mathcal{T}_{Q_{d}}=\stackrel{\phi}{d, r}^{*} \pi_{*} \mathcal{H} \operatorname{Hom}\left(\mathcal{E}_{d}, \mathcal{F}_{d}\right)=\left.\pi_{*} \mathcal{H o m}\left(\mathcal{E}_{d, r}, \mathcal{F}_{d, r}\right)\right|_{\AA_{d, r}}
\end{aligned}
$$


by Proposition 4.1 and the exact sequence

$$
0 \rightarrow \pi_{*} \mathcal{H o m}\left(\mathcal{E}_{d, r}, \mathcal{T}_{d, r}\right) \rightarrow \pi_{*} \mathcal{H o m}\left(\mathcal{E}_{d, r}, \mathcal{F}_{d, r}\right) \rightarrow \pi_{*} \mathcal{H o m}\left(\mathcal{E}_{d, r}, \bar{\theta}_{d, r}^{*} \mathcal{F}_{r}\right) \rightarrow 0
$$

obtained by applying $\pi_{*} \mathcal{H}$ om $\left(\mathcal{E}_{d, r},-\right)$ to the third column of the diagram (4.2). The dotted arrows in the third column are induced maps on the quotients. Since all rows and the middle column are exact, the third column is forced to be exact as well.

Using the identification

$$
\stackrel{\circ}{\theta}_{d, r}^{*} \mathcal{T}_{Q_{r}}=\stackrel{\circ}{\theta}_{d, r}^{*} \pi_{*} \mathcal{H o m}\left(\mathcal{E}_{r}, \mathcal{F}_{r}\right)=\left.\pi_{*} \mathcal{H o m}\left(\bar{\theta}_{d, r}^{*} \mathcal{E}_{r}, \bar{\theta}_{d, r}^{*} \mathcal{F}_{r}\right)\right|_{\AA_{d, r}}
$$

and comparing the third column with the short exact sequence

$$
0 \rightarrow \pi_{*} \mathcal{H o m}\left(\bar{\theta}_{d, r}^{*} \mathcal{E}_{r}, \bar{\theta}_{d, r}^{*} \mathcal{F}_{r}\right) \rightarrow \pi_{*} \mathcal{H o m}\left(\mathcal{E}_{d, r}, \bar{\theta}_{d, r}^{*} \mathcal{F}_{r}\right) \rightarrow \pi_{*} \mathcal{E} x t^{1}\left(\mathcal{T}_{d, r}, \bar{\theta}_{d, r}^{*} \mathcal{F}_{r}\right) \rightarrow 0
$$

obtained by applying $\pi_{*} \mathcal{H o m}\left(-, \bar{\theta}_{d, 0}^{*} \mathcal{F}_{0}\right)$ to the exact sequence $(4.1)$, we obtain a natural identification

$$
\mathcal{N}_{\AA_{d, r} / Q_{d}}=\left.\pi_{*} \mathcal{E} x t^{1}\left(\mathcal{T}_{d, r}, \bar{\theta}_{d, r}^{*} \mathcal{F}_{r}\right)\right|_{\AA_{d, r}}
$$

\section{The nORMAL BUndLES: THE GENERAL CASE}

In this technical section, we introduce and analyze the properties of a set of auxiliary schemes. These will be used in the final section to identify with and to derive the desired modular properties of the exceptional divisors created in the sequence of blowups $\widetilde{Q}_{d} \rightarrow Q_{d}$.

5.1. The schemes $Q_{d, r, l}$ and morphisms $\psi_{d, \underline{r}, l}$. Let $d>r>l \geq 0$, and we consider $Q_{d, r, l}:=Q_{d, r} \times_{Q_{r}} Q_{r, l}$. Let $\psi_{d, r, \underline{l}}: Q_{d, r, l} \rightarrow Q_{d, r}$ and $\psi_{\underline{d}, r, l}: Q_{d, r, l} \rightarrow$ $Q_{r, l}$ be the two projections. (Here the underscored subscript $\underline{l}$ in the map $\psi_{d, r, \underline{l}}$ indicates that the subscript $l$ shows up in the source $\left(Q_{d, r, l}\right)$ but not in the target $\left(Q_{d, r}\right)$. Ditto for $\underline{d}$, and for $\underline{r}$ below.) First of all, we see that $Q_{d, r, l}$ is smooth over $Q_{r, l}$ because $Q_{d, r}$ is smooth over $Q_{r}$. It follows that $Q_{d, r, l}$ is a nonsingular variety. Next, we will define a finite morphism $\psi_{d, \underline{r}, l}: Q_{d, r, l} \rightarrow Q_{d, l}$ based on the 
morphisms in the following diagram

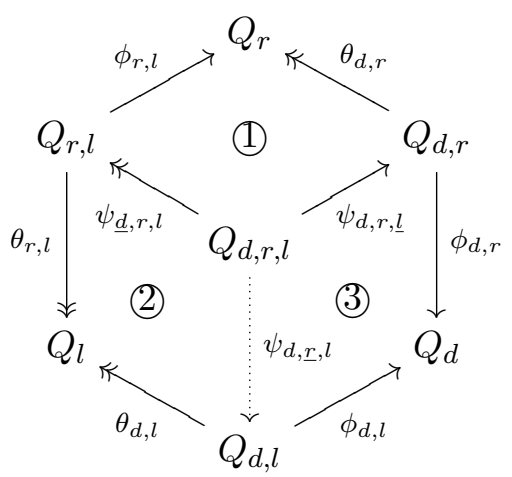

The parallelogram (1) is commutative by the definition of $Q_{d, r, l}$. On $\mathbb{P}^{1} \times Q_{d, r, l}$ we have two short exact sequences:

$$
\begin{aligned}
& 0 \rightarrow \bar{\psi}_{\underline{d}, r, l}^{*} \mathcal{E}_{r, l} \rightarrow \bar{\psi}_{\underline{d}, r, l}^{*} \bar{\theta}_{r, l}^{*} \mathcal{E}_{l} \rightarrow \bar{\psi}_{\underline{d}, r, l}^{*} \mathcal{T}_{r, l} \rightarrow 0 \\
& 0 \rightarrow \bar{\psi}_{d, r, \underline{l}}^{*} \mathcal{E}_{d, r} \rightarrow \bar{\psi}_{d, r, \underline{l}}^{*} \bar{\theta}_{d, r}^{*} \mathcal{E}_{r} \rightarrow \bar{\psi}_{d, r, \underline{l}}^{*} \mathcal{T}_{d, r} \rightarrow 0
\end{aligned}
$$

which are pullbacks of the universal exact sequences of $Q_{r, l}$ and $Q_{d, r}$ via $\bar{\psi}_{\underline{d}, r, l}$ and $\bar{\psi}_{d, r, \underline{l}}$ respectively. Note that $\bar{\psi}_{d, r, \underline{l}}^{*} \bar{\theta}_{d, r}^{*} \mathcal{E}_{r}=\bar{\psi}_{\underline{d}, r, l}^{*} \bar{\phi}_{r, l}^{*} \mathcal{E}_{r}=\bar{\psi}_{\underline{d}, r, l} \mathcal{E}_{r, l}$. Putting the two sequences together, we can form a commutative diagram as follows:

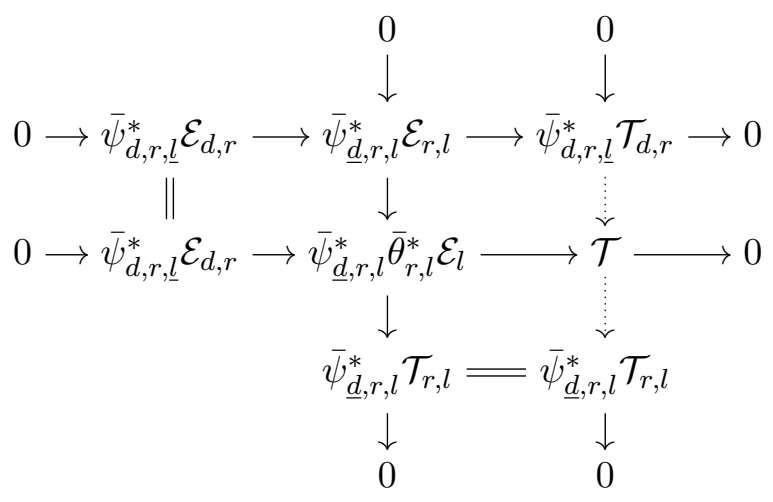

where $\mathcal{T}=\operatorname{Coker}\left(\bar{\psi}_{d, r, l}^{*} \mathcal{E}_{d, r} \rightarrow \bar{\psi}_{d, r, l}^{*} \mathcal{E}_{r, l} \rightarrow \bar{\psi}_{d, r, l}^{*} \bar{\theta}_{r, l}^{*} \mathcal{E}_{l}\right)$, and the dotted arrows are the induced maps on quotients. Since the upper two rows and the middle column are exact, the last column are forced to be exact as well. Note that $\bar{\psi}_{d, r, \underline{l}}^{*} \mathcal{T}_{d, r}$ and $\bar{\psi}_{\underline{d}, r, l}^{*} \mathcal{T}_{r, l}$ are both flat over $Q_{d, r, l}$ with rank 0 but with relative degree $d-r$ and $r-l$ respectively. Hence $\mathcal{T}$ is flat over $Q_{d, r, l}$ as well and is of rank 0 and of relative degree $d-l$. Thus the quotient $\bar{\psi}_{\underline{d}, r, l}^{*} \bar{\theta}_{r, l}^{*} \mathcal{E}_{l} \rightarrow \mathcal{T}$ from the middle row 
determines a $Q_{l}$-morphism

$$
\psi_{d, \underline{r}, l}: Q_{d, r, l} \rightarrow Q_{d, l}
$$

such that the pullback of the universal exact sequence of $Q_{d, l}$ is the middle row of (5.2), i.e., we have the following identifications:

$$
\begin{aligned}
0 \longrightarrow \bar{\psi}_{d, r, \underline{l}}^{*} \mathcal{E}_{d, r} \longrightarrow \bar{\psi}_{\underline{d}, r, l}^{*} \bar{\theta}_{r, l}^{*} \mathcal{E}_{l} \longrightarrow & \mathcal{T} \longrightarrow 0 \\
\| & \longrightarrow \bar{\psi}_{d, \underline{r}, l}^{*} \mathcal{E}_{d, l} \longrightarrow \bar{\psi}_{d, \underline{r}, l}^{*} \bar{\theta}_{d, l}^{*} \mathcal{E}_{l} \longrightarrow \bar{\psi}_{d, \underline{r}, l}^{*} \mathcal{T}_{d, l} \longrightarrow 0
\end{aligned}
$$

In particular, the identification $\mathcal{T}=\bar{\psi}_{d, \underline{r}, l}^{*} \mathcal{T}_{d, l}$ allows us to rewrite the third column of the diagram (5.2) as

$$
0 \rightarrow \bar{\psi}_{d, r, \underline{\underline{T}}}^{*} \mathcal{T}_{d, r} \rightarrow \bar{\psi}_{d, \underline{r}, l}^{*} \mathcal{T}_{d, l} \rightarrow \bar{\psi}_{\underline{d}, r, l}^{*} \mathcal{T}_{r, l} \rightarrow 0
$$

By the definition of $\psi_{d, \underline{r}, l}$, the parallelogram (2) in the diagram (5.1) automatically commutes. We also have

Proposition 5.1. The parallelogram (3) in diagram (5.1) commutes.

Proof. We need to show $\phi_{d, r} \psi_{d, r, \underline{l}}=\phi_{d, l} \psi_{d, \underline{r}, l}$. Because both $\phi_{d, r} \psi_{d, r, \underline{l}}$ and $\phi_{d, l} \psi_{d, \underline{r}, l}$ map into $Q_{d}$, by the universal property of the Quot scheme $Q_{d}$ (see [Shao11], Theorem 2.1), it suffices to show that there is an isomorphism $\left(\overline{\phi_{d, r} \psi_{d, r, \underline{l}}}\right) * \mathcal{F}_{d} \simeq$ $\left(\overline{\phi_{d, l} \psi_{d, \underline{r}, l}}\right) * \mathcal{F}_{d}$ that makes the following diagram commute:

$$
\begin{aligned}
V_{\mathbb{P}^{1} \times Q_{d, r, l}} & =\left(\overline{\phi_{d, r} \psi_{d, r, l}}\right)^{*} V_{\mathbb{P}^{1} \times Q_{d}} \rightarrow\left(\overline{\phi_{d, r} \psi_{d, r, \underline{l}}}\right)^{*} \mathcal{F}_{d} \\
\| & \\
V_{\mathbb{P}^{1} \times Q_{d, r, l}} & =\left(\overline{\phi_{d, l} \psi_{d, \underline{r}, l}}\right)^{*} V_{\mathbb{P}^{1} \times Q_{d}} \longrightarrow\left(\overline{\phi_{d, l} \psi_{d, \underline{r}, l}}\right)^{*} \mathcal{F}_{d}
\end{aligned}
$$

By the diagram (4.3), we have an exact sequence

$$
0 \rightarrow \mathcal{E}_{d, r} \rightarrow V_{\mathbb{P}^{1} \times Q_{d, r}} \rightarrow \bar{\phi}_{d, r}^{*} \mathcal{F}_{d} \rightarrow 0
$$

Replacing $r$ with $l$, we obtain another one

$$
0 \rightarrow \mathcal{E}_{d, l} \rightarrow V_{\mathbb{P}^{1} \times Q_{d, l}} \rightarrow \bar{\phi}_{d, l}^{*} \mathcal{F}_{d} \rightarrow 0
$$

Applying $\bar{\psi}_{d, r, \underline{l}}^{*}$ and $\bar{\psi}_{d, \underline{r}, l}^{*}$ to the above two exact sequences respectively, we obtain two exact sequences

$$
\begin{gathered}
0 \rightarrow \bar{\psi}_{d, r, \underline{l}}^{*} \mathcal{E}_{d, r} \rightarrow V_{\mathbb{P}^{1} \times Q_{d, r, l}} \rightarrow \bar{\psi}_{d, r, \underline{l}}^{*} \bar{\phi}_{d, r}^{*} \mathcal{F}_{d} \rightarrow 0 \\
0 \rightarrow \bar{\psi}_{d, \underline{r}, l}^{*} \mathcal{E}_{d, l} \rightarrow V_{\mathbb{P}^{1} \times Q_{d, r, l}} \rightarrow \bar{\psi}_{d, \underline{r}, l}^{*} \bar{\phi}_{d, l}^{*} \mathcal{F}_{d} \rightarrow 0
\end{gathered}
$$


Using the identification $\bar{\psi}_{d, r, \underline{l}}^{*} \mathcal{E}_{d, r}=\bar{\psi}_{d, \underline{r}, l}^{*} \mathcal{E}_{d, l}$ from the diagram (5.3), we obtain a commutative diagram

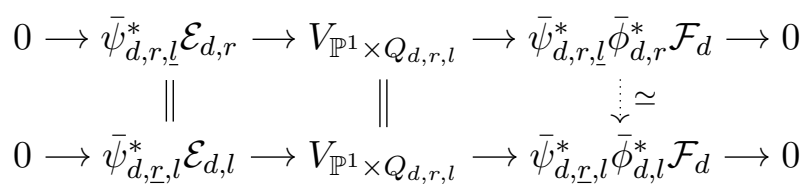

which induces an isomorphism $\bar{\psi}_{d, r, \underline{l}}^{*} \bar{\phi}_{d, r}^{*} \mathcal{F}_{d} \simeq \bar{\psi}_{d, \underline{r}, l}^{*} \bar{\phi}_{d, l}^{*} \mathcal{F}_{d}$ in the third column. This isomorphism is the desired one.

The map $\psi_{d, \underline{r}, l}$ is both proper and quasi-finite, hence it is a finite morphism.

Notation. For any integer $m \geq 1$, let $\Sigma_{m}$ be the set of all strictly decreasing sequences of nonnegative integers of length $m$ :

$$
\Sigma_{m}=\left\{\left(r_{1}, \cdots, r_{m}\right) \mid r_{1}>\cdots>r_{m}\right\}
$$

and let $\Sigma$ be the set of all strictly decreasing sequences of nonnegative integers of any finite length:

$$
\Sigma=\bigcup_{m=1}^{\infty} \Sigma_{m}
$$

For any sequence $\sigma=\left(r_{1}, \cdots, r_{m}\right) \in \Sigma$, the first term $r_{1}$ is called the leading term and is denoted as $\operatorname{lt}(\sigma)$. For any integer $r>\operatorname{lt}(\sigma)$, by $(r, \sigma)$ we mean the new sequence $\left(r, r_{1}, \cdots, r_{m}\right)$. The length of a sequence $\sigma$ is denoted by $|\sigma|$.

When we use a sequence of $\Sigma$ as sub-index, we would omit the parentheses. For example, if $\sigma=(8,5,3,1,0), \tau=(5,3,1,0)$ and $\lambda=(3,1,0)$, then the notations $Q_{\sigma}, Q_{8, \tau}$ and $Q_{8,5, \lambda}$ all mean the same thing: $Q_{8,5,3,1,0}$.

5.2. The schemes $P_{\sigma}$ and their properties. We now introduce a set of spaces $P_{\sigma}$ together with a set of coherent sheaves $\mathcal{X}_{\sigma}$ on $\mathbb{P}^{1} \times P_{\sigma}$, indexed by $\sigma \in \Sigma$. We will need a set of auxiliary spaces $R_{\sigma}$ indexed by $\sigma \in \Sigma$ with $|\sigma| \geq 2$. First, we define $P_{\sigma}$ for $\sigma \in \Sigma_{1}$. Suppose $\sigma=(d)$. In this case, we set

$$
P_{\sigma}=P_{d}:=Q_{d}, \quad \stackrel{\circ}{P}_{\sigma}=\stackrel{\circ}{P}_{d}:=\stackrel{\circ}{Q}_{d}, \quad \mathcal{X}_{\sigma}=\mathcal{X}_{d}:=\mathcal{F}_{d}
$$

We will denote by $\stackrel{\circ}{\mathcal{X}}_{d}$ the restriction of $\mathcal{X}_{d}$ on $\mathbb{P}^{1} \times \stackrel{\circ}{P}_{d}$. We know that $\stackrel{\circ}{\mathcal{X}}_{d}$ is locally free. 
Next we define $P_{\sigma}$ for $\sigma \in \Sigma_{2}$. Suppose $\sigma=(d, r), d>r$. We set

$$
R_{d, r}:=Q_{d, r} \times_{Q_{r}} P_{r}, \quad \stackrel{\circ}{R}_{d, r}:=Q_{d, r} \times_{Q_{r}} \stackrel{\circ}{P}_{r} \subset R_{d, r}
$$

Since $P_{r}=Q_{r}$ and $\stackrel{\circ}{P}_{r}=\stackrel{\circ}{Q}_{r}$, we actually have $R_{d, r}=Q_{d, r}$ and $\stackrel{\circ}{R}_{d, r}=\stackrel{\circ}{Q}_{d, r}$. Let $q_{d, r}$ and $p_{d, r}$ be the projections from $R_{d, r}$ to $Q_{d, r}$ and to $P_{r}$, respectively, and let $\stackrel{\circ}{q}_{d, r}$ and $\stackrel{\circ}{p}_{d, r}$ be the projections from $\stackrel{\circ}{R}_{d, r}$ to $Q_{d, r}$ and to $\stackrel{\circ}{P}_{r}$, respectively. We define $P_{d, r}$ to be a (relative) space of non-split extensions:

$$
P_{d, r}=\mathbb{P}\left(\pi_{*} \mathcal{E} x t^{1}\left(\stackrel{\bar{q}}{q} *, r_{d, r} \mathcal{T}_{d, r}, \bar{\rho}_{d, r}^{*} \mathcal{X}_{r}\right)\right)
$$

and let $a_{d, r}: P_{d, r} \rightarrow \stackrel{\circ}{R}_{d, r}$ be the structure morphism. We denote the universal extension on $\mathbb{P}^{1} \times P_{d, r}$ by

$$
0 \rightarrow\left(\bar{a}_{d, r}^{*} \stackrel{\bar{p}}{p} *, r^{*} \dot{\mathcal{X}}_{r}\right)(0,1) \rightarrow \mathcal{X}_{d, r} \rightarrow \bar{a}_{d, r}^{*} \stackrel{\bar{q}}{q}_{d, r}^{*} \mathcal{T}_{d, r} \rightarrow 0
$$

Let $\stackrel{\circ}{P}_{d, r} \subset P_{d, r}$ be the open subset defined as

$$
\stackrel{\circ}{P}_{d, r}:=\left\{x \in P_{d, r} \mid\left(\mathcal{X}_{d, r}\right)_{x} \text { is locally free }\right\}
$$

We denote by $\stackrel{\circ}{\mathcal{X}}_{d, r}$ the restriction of $\mathcal{X}_{d, r}$ to $\mathbb{P}^{1} \times \stackrel{\circ}{P}_{d, r}$. Then $\stackrel{\circ}{\mathcal{X}}_{d, r}$ is locally free, and $P_{d, r}$ can be considered as a $Q_{d}$-scheme through the composition $P_{d, r} \rightarrow \stackrel{\circ}{R}_{d, r} \rightarrow$ $Q_{d, r} \rightarrow Q_{d}$. So we have defined $P_{\sigma}, \mathcal{X}_{\sigma}, R_{\sigma}$, etc., for any $\sigma \in \Sigma$ with $|\sigma|=2$. In the following, we will define $P_{\sigma}, \mathcal{X}_{\sigma}$, etc., for any $\sigma \in \Sigma$ with $|\sigma| \geq 3$ inductively.

Assume that, for each $\sigma \in \Sigma_{m}$ for some $m \geq 2$, the space $P_{\sigma}$ of non-split extensions is defined and the sheaf $\mathcal{X}_{\sigma}$ is the middle term from the universal extension on $\mathbb{P}^{1} \times P_{\sigma}$. Assume also that a morphism $P_{\sigma} \rightarrow Q_{l}(l=\operatorname{lt}(\sigma))$ has been specified so that $P_{\sigma}$ can be considered as a $Q_{l}$-scheme.

Let $\sigma \in \Sigma_{m+1}, d=\operatorname{lt}(\sigma), \tau$ be the sequence formed from $\sigma$ by removing the leading term $d$, and $r=\operatorname{lt}(\tau)$. So $\sigma=(d, \tau)=(d, r, \cdots)$. By induction hypothesis, the space $P_{\tau}$ of non-split extensions is defined and is a $Q_{r}$-scheme. We set

$$
R_{\sigma}:=Q_{d, r} \times_{Q_{r}} P_{\tau}, \quad \stackrel{\circ}{R}_{\sigma}:=Q_{d, r} \times_{Q_{r}} \stackrel{\circ}{\tau}_{\tau} \subset R_{\sigma}
$$

Let $q_{\sigma}$ and $p_{\sigma}$ be the two projections from $R_{\sigma}$ to $Q_{d, r}$ and to $P_{\tau}$, respectively, and let $\stackrel{\circ}{q}_{\sigma}$ and $\stackrel{\circ}{p}_{\sigma}$ be the two projections from $\stackrel{\circ}{R}_{\sigma}$ to $Q_{d, r}$ and to $\stackrel{\circ}{P}_{\tau}$, respectively. We define $P_{\sigma}=P_{d, \tau}$ to be a space of non-split extensions over $\stackrel{\circ}{R}_{\sigma}$ by

$$
P_{\sigma}:=\mathbb{P}\left(\pi_{*} \mathcal{E} x t^{1}\left(\overline{\bar{q}}_{\sigma}^{*} \mathcal{T}_{d, r}, \overline{\bar{p}}_{\sigma}^{*} \dot{\mathcal{X}}_{\tau}\right)\right)
$$


and let $a_{\sigma}: P_{\sigma} \rightarrow \stackrel{\circ}{R}_{\sigma}$ be the structure morphism. We denote the universal extension on $\mathbb{P}^{1} \times P_{\sigma}$ by

$$
0 \rightarrow\left(\bar{a}_{\sigma}^{*} \overline{\bar{p}}_{\sigma}^{*} \stackrel{\circ}{\mathcal{X}}_{\tau}\right)(0,1) \rightarrow \mathcal{X}_{\sigma} \rightarrow \bar{a}_{\sigma}^{*} \overline{\bar{q}}_{\sigma}^{*} \mathcal{T}_{d, r} \rightarrow 0
$$

$P_{\sigma}$ can be considered as a $Q_{d}$-scheme through $P_{\sigma} \rightarrow \stackrel{\circ}{R}_{\sigma} \rightarrow Q_{d, r} \rightarrow Q_{d}$. We define the open subset $\stackrel{\circ}{P}_{\sigma} \subset P_{\sigma}$ as

$$
\stackrel{\circ}{P}_{\sigma}:=\left\{x \in P_{\sigma} \mid\left(\mathcal{X}_{\sigma}\right)_{x} \text { is locally free }\right\}
$$

Then, $\dot{\mathcal{X}}_{\sigma}$, the restriction of $\mathcal{X}_{\sigma}$ on $\mathbb{P}^{1} \times \stackrel{\circ}{P}_{\sigma}$ is locally free. By induction, we have defined $P_{\sigma}, \mathcal{X}_{\sigma}, R_{\sigma}$, etc., for all $\sigma \in \Sigma$.

Lemma 5.2. For each $\sigma$,

(1) $\mathcal{X}_{\sigma}$ is flat over $\mathbb{P}_{\sigma}$ with relative degree $\operatorname{lt}(\sigma)$.

(2) $R^{1} \pi_{*}\left(\mathcal{X}_{\sigma}(-1)\right)=0$.

The closed subset $P_{\sigma} \backslash \stackrel{\circ}{P}_{\sigma}$ of $P_{\sigma}$ has a sequence of nested closed subschemes

$$
\varnothing=Y_{d, r, \tau} \subset Y_{d, r+1, \tau} \subset \cdots \subset Y_{d, d-1, \tau}=P_{\sigma} \backslash \stackrel{\circ}{P}_{\sigma}
$$

where $Y_{d, e, \tau}=\left\{x \in P_{\sigma} \mid \operatorname{deg}\left(\left(\mathcal{X}_{\sigma}\right)_{x}^{\mathbf{t}}\right) \geq d-e\right\}$ and the subscheme structure on $Y_{d, e, \tau}$ is defined by (3.3). We set $\stackrel{\circ}{Y, e, \tau}_{d,=} Y_{d, e, \tau} \backslash Y_{d, e-1, \tau}$ for $e=r+1, \cdots, d-1$. That is, $\stackrel{\circ}{Y}_{d, e, \tau}=\left\{x \in P_{\sigma} \mid \operatorname{deg}\left(\left(\mathcal{X}_{\sigma}\right)_{x}^{\mathbf{t}}\right)=d-e\right\}$.

Lemma 5.3. The space $P_{\sigma}$ is nonsingular for any $\sigma \in \Sigma$.

Proof. We prove it by induction on $|\sigma|$. When $|\sigma|=1$, say $\sigma=(d)$ for some $d$, we have $P_{\sigma}=P_{d}=Q_{d}$. So this case is obvious since $Q_{d}$ is nonsingular. Assume that the statement holds true for all $\sigma \in \Sigma_{m}$ for some $m$. Let $\sigma \in \Sigma_{m+1}$ and suppose $\sigma=(d, \tau)$ where $\tau \in \Sigma_{m}$. By definition, $P_{\sigma}=P_{d, \tau}$ is a projective bundle over $Q_{d, r} \times_{Q_{r}} \stackrel{\circ}{P}_{\tau}$ where $r=\operatorname{lt}(\tau)$. Hence $P_{\sigma}$ is smooth over $Q_{d, r} \times_{Q_{r}} \stackrel{\circ}{P}_{\tau}$. We know that $Q_{d, r}$ is smooth over $Q_{r}$, hence $Q_{d, r} \times_{Q_{r}} \stackrel{\circ}{P}_{\tau}$ is smooth over $\stackrel{\circ}{P}_{\tau}$. Since smoothness is transitive, $P_{\sigma}$ is smooth over $\stackrel{\circ}{P}_{\tau}$. By induction hypothesis, $P_{\tau}$ is nonsingular, and so is $\stackrel{\circ}{P}_{\tau}$ and hence $P_{\sigma}$ is nonsingular. This completes the proof. 
Let $\sigma \in \Sigma, l=\operatorname{lt}(\sigma)$ and let $d>r>l$. We define a morphism $\phi_{d, r, \sigma}: R_{d, r, \sigma} \rightarrow$ $P_{d, \sigma}$ using the following commutative diagram

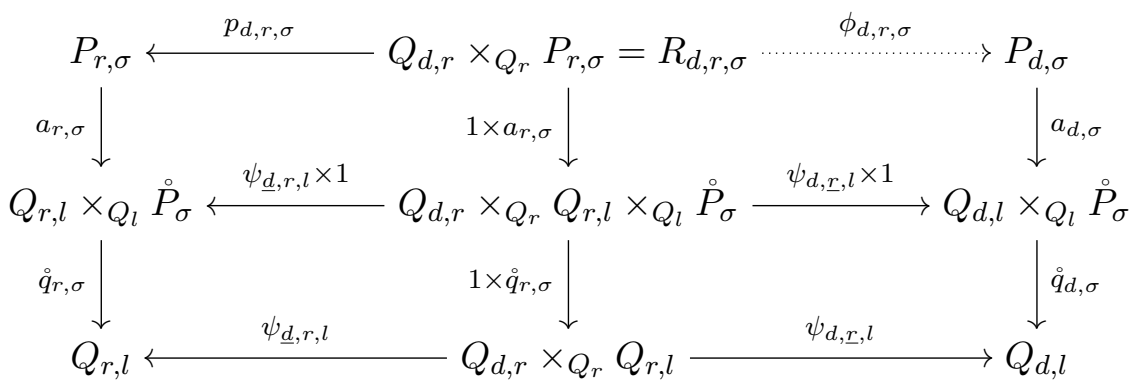

By the base change property, $R_{d, r, \sigma}$ is a projective bundle over $Q_{d, r} \times_{Q_{r}} \stackrel{\circ}{R}, \sigma=$ $Q_{d, r} \times_{Q_{r}} Q_{r, l} \times_{Q_{l}} \stackrel{\circ}{P}_{\sigma}$

$$
R_{d, r, \sigma}=\left(Q_{d, r} \times_{Q_{r}} \stackrel{\circ}{R}_{r, \sigma}\right) \times_{\dot{R}_{r, \sigma}} P_{r, \sigma}=\mathbb{P}\left(\left(\psi_{\underline{d}, r, l} \times 1\right)^{*} \pi_{*} \mathcal{E} x t^{1}\left(\overline{\stackrel{s}{q}}_{r, \sigma}^{*} \mathcal{T}_{r, l},{\stackrel{\bar{p}^{*}}{r, \sigma}}_{\mathcal{X}_{\sigma}}\right)\right)
$$

with $\mathcal{O}_{R_{d, r, \sigma}}(1)=p_{d, r, \sigma}^{*} \mathcal{O}_{P_{r, \sigma}}(1)$ and structure morphism $1 \times a_{r, \sigma}$. On $\mathbb{P}^{1} \times R_{d, r, \sigma}$, we have a commutative diagram of sheaves

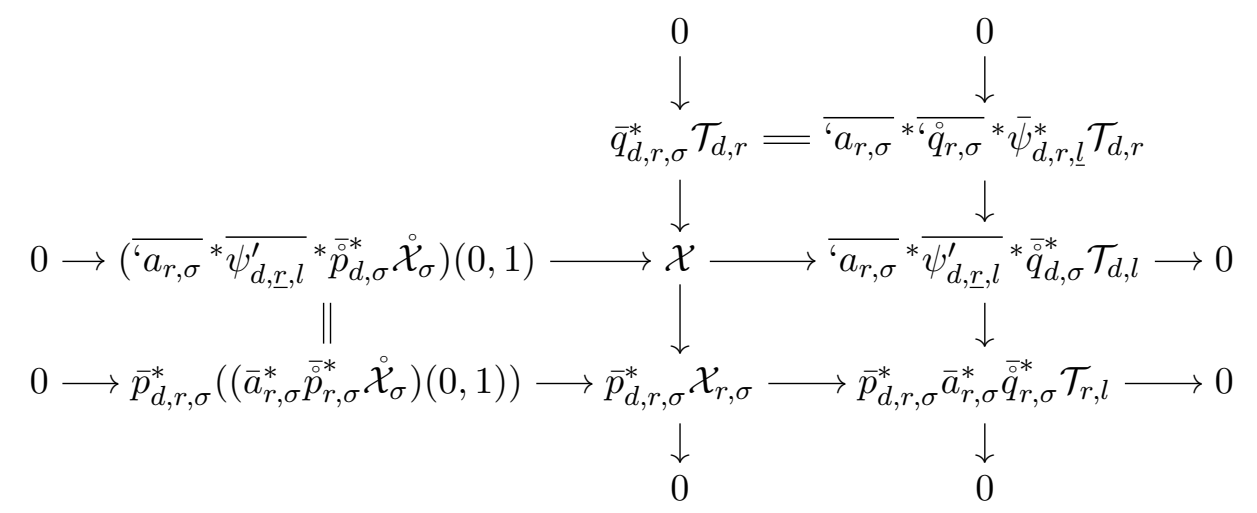

where

- for short notations, we have set ' $a_{r, \sigma}:=1 \times a_{r, \sigma},{ }^{\prime} \stackrel{\leftrightarrow}{r, \sigma}_{r}:=1 \times q_{r, \sigma}, \psi_{d, \underline{r}, l}^{\prime}:=$ $\psi_{d, \underline{r}, l} \times 1$

- the last row is the pullback of the universal extension of $P_{r, \sigma}$ via $\bar{p}_{d, r, \sigma}$ : $\mathbb{P}^{1} \times R_{d, r, \sigma} \rightarrow \mathbb{P}^{1} \times P_{r, \sigma}$,

- the last column is the pullback of the exact sequence of torsion sheaves

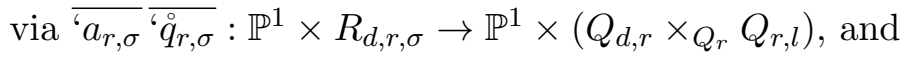

- $\mathcal{X}$ is the fiber product $\left(\bar{p}_{d, r, \sigma}^{*} \mathcal{X}_{r, \sigma}\right) \times_{\left(\bar{p}_{d, r, \sigma}^{*} \bar{a}_{r, \sigma}^{*} \bar{q}_{r, \sigma}^{*} \mathcal{T}_{r, l}\right)}\left({ }^{{ }^{a_{r, \sigma}}} * \bar{\psi}_{d, \underline{r}, l}^{\prime}{ }^{*} \bar{q}_{r, \sigma}^{*} \mathcal{T}_{d, l}\right)$ in the category of coherent sheaves. 
Recall that the universal extension of $P_{d, \sigma}$ is the exact sequence

$$
0 \rightarrow\left(\bar{a}_{d, \sigma}^{*} \overline{\dot{p}}_{d, \sigma}^{*} \dot{\mathcal{X}}_{\sigma}\right)(0,1) \rightarrow \mathcal{X}_{d, \sigma} \rightarrow \bar{a}_{d, \sigma}^{*} \overline{\bar{q}}_{d, \sigma}^{*} \mathcal{T}_{d, l} \rightarrow 0
$$

on $\mathbb{P}^{1} \times P_{d, \sigma}$. By Theorem 2.2 , the middle row determines a $\left(Q_{d, l} \times_{Q_{l}} \stackrel{\circ}{P}_{\sigma}\right)$ morphism

$$
\phi_{d, r, \sigma}: R_{d, r, \sigma} \rightarrow P_{d, \sigma}
$$

such that there are isomorphisms $\mathcal{O}_{R_{d, r, \sigma}}(1) \simeq \phi_{d, r, \sigma}^{*} \mathcal{O}_{P_{d, \sigma}}(1)$ and $\mathcal{X} \simeq \bar{\phi}_{d, r, \sigma}^{*} \mathcal{X}_{d, \sigma}$ that make the following diagram commute:

$$
\begin{aligned}
& 0 \longrightarrow\left(\overline{{ }^{6} a_{r, \sigma}} * \overline{\psi_{d, \underline{r}, l}^{\prime}} * \bar{p}_{d, \sigma}^{*} \dot{\mathcal{X}}_{\sigma}\right)(0,1) \longrightarrow \mathcal{X} \longrightarrow \overline{ }^{6} a_{r, \sigma} *{\overline{\psi_{d, \underline{r}, l}^{\prime}}}^{\prime}{ }^{\bar{q}_{d, \sigma}^{*}} \mathcal{T}_{d, l} \longrightarrow 0 \\
& \downarrow \simeq \quad \downarrow \simeq \quad \| \\
& 0 \longrightarrow \bar{\phi}_{d, r, \sigma}^{*}\left(\left(\bar{a}_{d, \sigma}^{*}{ }^{\bar{p}^{*} *}{ }_{d, \sigma}^{\dot{\mathcal{X}}_{\sigma}}\right)(0,1)\right) \longrightarrow \bar{\phi}_{d, r, \sigma}^{*} \mathcal{X}_{d, \sigma} \longrightarrow \bar{\phi}_{d, r, \sigma}^{*} \bar{a}_{d, \sigma}^{*} \overline{\bar{q}}_{d, \sigma}^{*} \mathcal{T}_{d, l} \longrightarrow 0
\end{aligned}
$$

where the first row is the middle row of diagram (5.6) and the second row is the pullback of the universal extension (5.7) of $P_{d, \sigma}$ via $\bar{\phi}_{d, r, \sigma}: \mathbb{P}^{1} \times R_{d, r, \sigma} \rightarrow \mathbb{P}^{1} \times P_{d, \sigma}$. For simplicity, we make identifications

$$
\mathcal{O}_{R_{d, r, \sigma}}(1)=\phi_{d, r, \sigma}^{*} \mathcal{O}_{P_{d, \sigma}}(1), \quad \mathcal{X}=\bar{\phi}_{d, r, \sigma}^{*} \mathcal{X}_{d, \sigma}
$$

Let $\stackrel{\circ}{\phi}_{d, r, \sigma}: \stackrel{\circ}{R}_{d, r, \sigma} \rightarrow P_{d, \sigma}$ be the restriction of $\phi_{d, r, \sigma}$ to $\stackrel{\circ}{R}_{d, r, \sigma}$. The restriction of the middle column of diagram (5.6) to $\mathbb{P}^{1} \times \stackrel{\circ}{R}_{d, r, \sigma}$ is the exact sequence

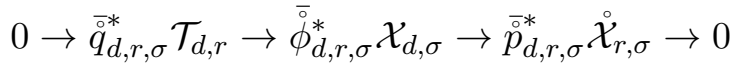

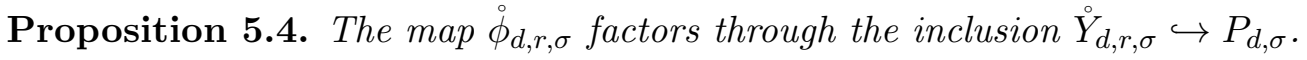

Proof. Taking the dual of the sequence (5.9), we obtain $\left(\bar{\phi}_{d, r, \sigma}^{*} \mathcal{X}_{d, \sigma}\right)^{\varepsilon} \simeq\left(\bar{q}_{d, r, \sigma}^{*} \mathcal{T}_{d, r}\right)^{\varepsilon}$. Since $\left(\overline{\bar{\phi}}_{d, r, \sigma}^{*} \mathcal{X}_{d, \sigma}\right)^{\varepsilon}=\overline{\bar{\phi}}_{d, r, \sigma}^{*}\left(\mathcal{X}_{d, \sigma}^{\varepsilon}\right)$, we have that $\overline{\bar{\phi}}_{d, r, \sigma}^{*}\left(\mathcal{X}_{d, \sigma}^{\varepsilon}\right)$ is flat over $\stackrel{\circ}{R}_{d, r, \sigma}$ with relative degree $d-r$. By Proposition 3.4, the map $\dot{\phi}_{d, r, \sigma}$ factors through the inclusion $\stackrel{\circ}{\text { d,r, }}_{\hookrightarrow} \hookrightarrow P_{d, \sigma}$.

We denote by $\stackrel{\circ}{\varphi}_{d, r, \sigma}: \stackrel{\circ}{R}_{d, r, \sigma} \rightarrow \stackrel{\circ}{Y}_{d, r, \sigma}$ the map factored out from $\stackrel{\circ}{\phi}_{d, r, \sigma}$.

Proposition 5.5. The morphism $\stackrel{\circ}{\varphi, r, \sigma}: \stackrel{\circ}{R}_{d, r, \sigma} \rightarrow \stackrel{\circ}{Y}_{d, r, \sigma}$ is an isomorphism.

Proof. We prove by constructing an inverse of $\stackrel{\circ}{\varphi}_{d, r, \sigma}$. Let $i: \stackrel{\circ}{Y}_{d, r, \sigma} \hookrightarrow P_{d, \sigma}$ be the inclusion map. The pullback of the universal extension (5.7) via $i$ is the exact sequence

$$
0 \rightarrow \bar{i}^{*}\left(\left(\bar{a}_{d, \sigma}^{*} \stackrel{\bar{p}}{p} *, \sigma^{*} \dot{\mathcal{X}}_{\sigma}\right)(0,1)\right) \rightarrow \bar{i}^{*} \mathcal{X}_{d, \sigma} \rightarrow \bar{i}^{*} \bar{a}_{d, \sigma}^{*} \stackrel{\bar{q}}{d, \sigma} *^{*} \mathcal{T}_{d, l} \rightarrow 0
$$


on $\mathbb{P}^{1} \times \stackrel{\circ}{Y}_{d, r, \sigma}$. Taking dual, we obtain a long exact sequence

$$
0 \rightarrow\left(\bar{i}^{*} \mathcal{X}_{d, \sigma}\right)^{\vee} \rightarrow\left(\bar{i}^{*}\left(\left(\bar{a}_{d, \sigma}^{*} \overline{\bar{p}}_{d, \sigma}^{*} \dot{\mathcal{X}}_{\sigma}\right)(0,1)\right)\right)^{\vee} \rightarrow\left(\bar{i}^{*} \bar{a}_{d, \sigma}^{*} \overline{\bar{q}}_{d, \sigma}^{*} \mathcal{T}_{d, l}\right)^{\varepsilon} \rightarrow\left(\bar{i}^{*} \mathcal{X}_{d, \sigma}\right)^{\varepsilon} \rightarrow 0
$$

We break it into two short exact sequences

$$
\begin{gathered}
0 \rightarrow\left(\bar{i}^{*} \mathcal{X}_{d, \sigma}\right)^{\vee} \rightarrow\left(\bar{i}^{*}\left(\left(\bar{a}_{d, \sigma}^{*} \overline{\bar{p}}_{d, \sigma}^{*} \dot{\mathcal{X}}_{\sigma}\right)(0,1)\right)\right)^{\vee} \rightarrow \mathcal{T} \rightarrow 0 \\
0 \rightarrow \mathcal{T} \rightarrow\left(\bar{i}^{*} \bar{a}_{d, \sigma}^{*} \bar{q}_{d, \sigma}^{*} \mathcal{T}_{d, l}\right)^{\varepsilon} \rightarrow\left(\bar{i}^{*} \mathcal{X}_{d, \sigma}\right)^{\varepsilon} \rightarrow 0
\end{gathered}
$$

We have $\left(\bar{i}^{*} \mathcal{X}_{d, \sigma}\right)^{\varepsilon}=\bar{i}^{*}\left(\mathcal{X}_{d, \sigma}^{\varepsilon}\right)$ and $\left(\bar{i}^{*} \bar{a}_{d, \sigma}^{*} \overline{\bar{q}}_{d, \sigma}^{*} \mathcal{T}_{d, l}\right)^{\varepsilon}=\bar{i}^{*} \bar{a}_{d, \sigma}^{*} \overline{\bar{q}}_{d, \sigma}^{*}\left(\mathcal{T}_{d, l}^{\varepsilon}\right)$. By Proposition $3.4, \bar{i}^{*}\left(\mathcal{X}_{d, \sigma}^{\varepsilon}\right)$ is flat over $\stackrel{\circ}{y, r, \sigma}$ with relative degree $d-r$. Since $\mathcal{T}_{d, l}^{\varepsilon}$ is also flat over $Q_{d, l}$ with relative degree $d-l$, we have $\mathcal{T}$ is flat over $\dot{\Upsilon}_{d, r, \sigma}$ with relative degree $r-l$. We also know that both $\left(\bar{i}^{*} \bar{a}_{d, \sigma}^{*} \overline{\bar{q}}_{d, \sigma}^{*} \mathcal{T}_{d, l}\right)^{\varepsilon}$ and $\left(\bar{i}^{*} \mathcal{X}_{d, \sigma}\right)^{\varepsilon}$ are torsion, hence $\mathcal{T}$ is also torsion. Since the middle term of the first sequence is locally free and the last term is flat over $\stackrel{\circ}{Y}_{d, r, \sigma}$, the first term, $\left(\bar{i}^{*} \mathcal{X}_{d, \sigma}\right)^{\vee}$, is locally free as well.

Now dualizing both of the above sequences, we obtain another two exact sequences

$$
\begin{gathered}
0 \rightarrow \bar{i}^{*}\left(\left(\bar{a}_{d, \sigma}^{*} \bar{p}_{d, \sigma}^{*} \dot{\mathcal{X}}_{\sigma}\right)(0,1)\right) \rightarrow\left(\bar{i}^{*} \mathcal{X}_{d, \sigma}\right)^{\vee \vee} \rightarrow \mathcal{T}^{\varepsilon} \rightarrow 0 \\
0 \rightarrow\left(\bar{i}^{*} \mathcal{X}_{d, \sigma}\right)^{\varepsilon \varepsilon} \rightarrow \bar{i}^{*} \bar{a}_{d, \sigma}^{*} \overline{\bar{q}}_{d, \sigma}^{*} \mathcal{T}_{d, l} \rightarrow \mathcal{T}^{\varepsilon} \rightarrow 0
\end{gathered}
$$

The Quot scheme $Q_{d, l}$ has a universal quotient $\bar{\theta}_{d, l}^{*} \mathcal{E}_{l} \rightarrow \mathcal{T}_{d, l}$. Applying $\bar{i}^{*} \bar{a}_{d, \sigma}^{*} \stackrel{\bar{q}}{d, \sigma}^{*}$ to this quotient, we obtain a quotient $\bar{i}^{*} \bar{a}_{d, \sigma}^{*}{ }_{\bar{q}}^{\bar{q}_{d, \sigma}^{*}} \bar{\theta}_{d, l}^{*} \mathcal{E}_{l} \rightarrow \bar{i}^{*} \bar{a}_{d, \sigma}^{*} \bar{q}_{d, \sigma}^{*} \mathcal{T}_{d, l}$. Its composition with the quotient $\bar{i}^{*} \bar{a}_{d, \sigma}^{*} \overline{\bar{q}}_{d, \sigma}^{*} \mathcal{T}_{d, l} \rightarrow \mathcal{T}^{\varepsilon}$ from the sequence (5.11) yields a quotient

We form a commutative diagram

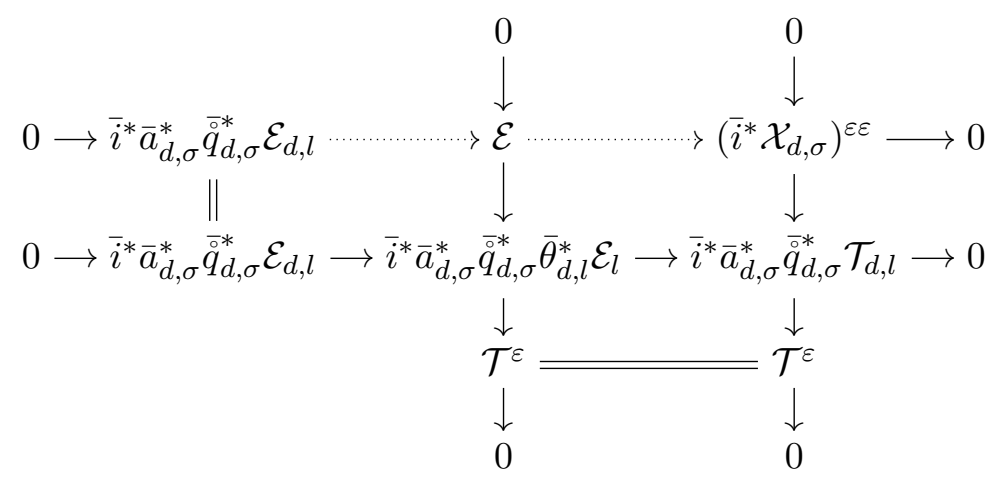


where the last column is the sequence (5.11), the middle row is the pullback of the universal exact sequence of $Q_{d, l}$ via $\overline{\bar{q}}_{d, \sigma} \bar{a}_{d, \sigma} \bar{i}: \mathbb{P}^{1} \times \stackrel{\circ}{Y, r, \sigma} \rightarrow \mathbb{P}^{1} \times Q_{d, l}$, and $\mathcal{E}$ is defined to be the kernel of the composition $\bar{i}^{*} \bar{a}_{d, \sigma}^{*} \overline{\bar{q}}_{d, \sigma}^{*} \bar{\theta}_{d, l}^{*} \mathcal{E}_{l} \rightarrow \bar{i}^{*} \bar{a}_{d, \sigma}^{*} \overline{\bar{q}}_{d, \sigma}^{*} \mathcal{T}_{d, l} \rightarrow \mathcal{T}^{\varepsilon}$. The dotted arrows in the first row are the induced maps. Since $\mathcal{T}^{\varepsilon}$ is torsion and flat over $\stackrel{\circ}{d, r, \sigma}$ with relative degree $r-l$, the quotient $\bar{i}^{*} \bar{a}_{d, \sigma}^{*} \overline{\bar{q}}_{d, \sigma}^{*} \bar{\theta}_{d, l}^{*} \mathcal{E}_{l} \rightarrow \mathcal{T}^{\varepsilon}$ from the middle column induces a $Q_{l}$-morphism $\lambda: \stackrel{\circ}{Y}_{d, r, \sigma} \rightarrow Q_{r, l}$ such that the pullback of the universal exact sequence of $Q_{r, l}$ via $\bar{\lambda}$ is the same as the middle column:

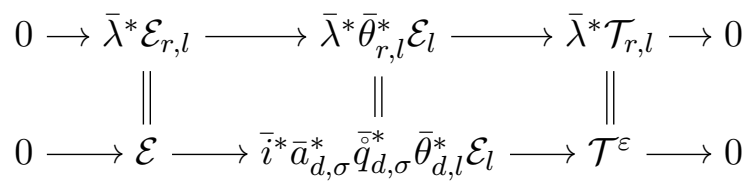

We have a morphism $\phi_{r, l}: Q_{r, l} \rightarrow Q_{r}$ and an identification $\mathcal{E}_{r, l}=\bar{\phi}_{r, l}^{*} \mathcal{E}_{r}$ as in diagram (4.3). Thus we have an identification

$$
\mathcal{E}=\bar{\lambda}^{*} \mathcal{E}_{r, l}=\bar{\lambda}^{*} \bar{\phi}_{r, l}^{*} \mathcal{E}_{r}
$$

and we can rewrite the first row as

$$
0 \rightarrow \bar{i}^{*} \bar{a}_{d, \sigma}^{*} \overline{\bar{q}}_{d, \sigma}^{*} \mathcal{E}_{d, l} \rightarrow \bar{\lambda}^{*} \bar{\phi}_{r, l}^{*} \mathcal{E}_{r} \rightarrow\left(\bar{i}^{*} \mathcal{X}_{d, \sigma}\right)^{\varepsilon \varepsilon} \rightarrow 0
$$

Since $\left(\bar{i}^{*} \mathcal{X}_{d, \sigma}\right)$ is is torsion and flat over $\stackrel{\circ}{Y}_{d, r, \sigma}$ with relative degree $d-r$, the above sequence induces a morphism $\mu: \stackrel{\circ}{Y}_{d, r, \sigma} \rightarrow Q_{d, r}$ such that the pullback of the universal exact sequence of $Q_{d, r}$ via $\bar{\mu}$ is the same as the above sequence:

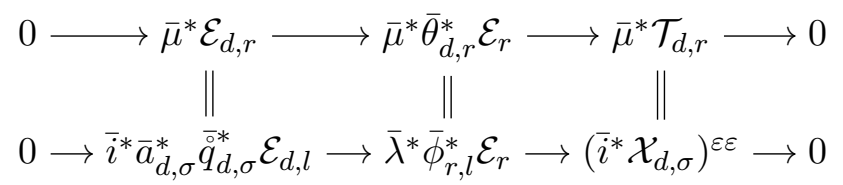

Using the identification $\mathcal{T}^{\varepsilon}=\bar{\lambda}^{*} \mathcal{T}_{r, l}$ from sequence (5.12), we can rewrite the sequence (5.10) as

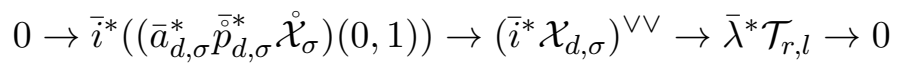

By Theorem 2.2, the above exact sequence determines a morphism $\nu: \dot{\circ}_{d, r, \sigma} \rightarrow$ $P_{r, \sigma}$ such that the pullback of the universal extension of $P_{r, \sigma}$ is the same as the 
above sequence:

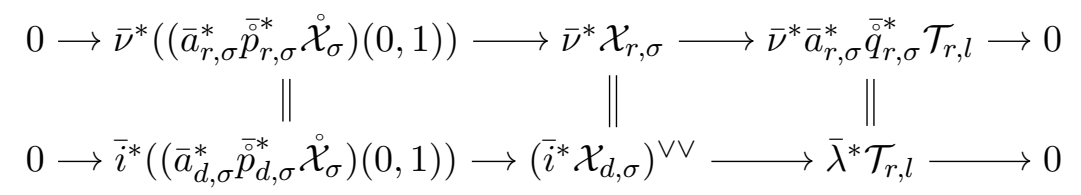

But because the middle term $\left(\bar{i}^{*} \mathcal{X}_{d, \sigma}\right)^{\vee \vee}$ is locally free, the map $\nu$ actually maps $\stackrel{\circ}{Y}_{d, r, \sigma}$ into $\stackrel{\circ}{P}_{r, \sigma}$. So we obtain a morphism

$$
\mu \times \nu: \stackrel{\circ}{Y}_{d, r, \sigma} \rightarrow Q_{d, r} \times_{Q_{r}} \stackrel{\circ}{P}_{r, \sigma}:=\stackrel{\circ}{R}_{d, r, \sigma}
$$

It is now routine to check that $\mu \times \nu$ is the inverse of $\stackrel{\circ}{\varphi}_{d, r, \sigma}: \stackrel{\circ}{R}_{d, r, \sigma} \rightarrow \stackrel{\circ}{Y}_{d, r, \sigma}$, and this complete the proof.

The above proposition shows that $\dot{\phi}_{d, r, \sigma}$ is an embedding. So we can identify $\stackrel{\circ}{R}_{d, r, \sigma}$ with the subscheme $\stackrel{\circ}{Y}_{d, r, \sigma}$ of $P_{d, \sigma}$ and identify $\stackrel{\circ}{d, r, \sigma}_{\mathcal{X}}$ with the inclusion map. We also have $\bar{\phi}_{d, r, \sigma}^{*} \mathcal{X}_{d, \sigma}=\mathcal{X}_{\AA_{d, r, \sigma}}$. So the exact sequence (5.9) can be rewritten as:

$$
0 \rightarrow \overline{\bar{q}}_{d, r, \sigma}^{*} \mathcal{T}_{d, r} \rightarrow \overline{\bar{\phi}}_{d, r, \sigma}^{*} \mathcal{X}_{d, \sigma} \rightarrow \overline{\bar{p}}_{d, r, \sigma}^{*} \dot{\circ}_{r, \sigma} \rightarrow 0
$$

Since $R_{d, r, \sigma}$ and $P_{d, \sigma}$ are both nonsingular, we can talk about their tangent bundles as well as the normal bundle of the embedding $\stackrel{\circ}{\phi}_{d, r, \sigma}: \stackrel{\circ}{R}_{d, r, \sigma} \hookrightarrow P_{d, \sigma}$.

Proposition 5.6. The normal bundle $\mathcal{N}_{\stackrel{\circ}{R}_{d, r, \sigma} / P_{d, \sigma}}$ of the embedding $\stackrel{\circ}{\phi}, r, \sigma: \stackrel{\circ}{R}_{d, r, \sigma} \hookrightarrow$ $P_{d, \sigma}$ is isomorphic to $\pi_{*} \mathcal{E} x t^{1}\left(\overline{\bar{q}}_{d, r, \sigma}^{*} \mathcal{T}_{d, r}, \overline{\bar{p}}_{d, r, \sigma}^{*} \dot{\mathcal{X}}_{r, \sigma}\right)$.

Proof. Since $P_{d, \sigma}$ is a projective bundle over $\stackrel{\circ}{R}_{d, \sigma}$, the relative cotangent bundle $\Omega_{P_{d, \sigma} / \AA^{R}, \sigma}$ fits into the following exact sequence

$$
0 \rightarrow \Omega_{P_{d, \sigma} / \stackrel{\circ}{R}_{d, \sigma}} \rightarrow\left(a_{d, \sigma}^{*} \pi_{*} \mathcal{E} x t^{1}\left(\overline{\bar{q}}_{d, \sigma}^{*} \mathcal{T}_{d, l}, \bar{p}_{d, \sigma}^{*} \dot{\mathcal{X}}_{\sigma}\right)\right)^{\vee}(-1) \rightarrow \mathcal{O}_{P_{d, \sigma}} \rightarrow 0
$$

Pulling the sequence back to $R_{d, r, \sigma}$ via $\phi_{d, r, \sigma}$, we obtain an exact sequence

$0 \rightarrow \phi_{d, r, \sigma}^{*} \Omega_{P_{d, \sigma} / \stackrel{\circ}{R}_{d, \sigma}} \rightarrow\left(\phi_{d, r, \sigma}^{*} a_{d, \sigma}^{*} \pi_{*} \mathcal{E} x t^{1}\left(\stackrel{\bar{q}}{q}_{d, \sigma}^{*} \mathcal{T}_{d, l}, \overline{\bar{p}}_{d, \sigma}^{*} \stackrel{\circ}{\mathcal{X}}_{\sigma}\right)\right)^{\vee}(-1) \rightarrow \phi_{d, r, \sigma}^{*} \mathcal{O}_{P_{d, \sigma}} \rightarrow 0$

We can rewrite $\phi_{d, r, \sigma}^{*} a_{d, \sigma}^{*} \pi_{*} \mathcal{E} x t^{1}\left(\overline{\bar{q}}_{d, \sigma}^{*} \mathcal{T}_{d, l}, \overline{\bar{p}}_{d, \sigma}^{*} \dot{\mathcal{X}}_{\sigma}\right)$ as

$$
\begin{aligned}
& \phi_{d, r, \sigma}^{*} a_{d, \sigma}^{*} \pi_{*} \mathcal{E} x t^{1}\left(\overline{\bar{q}}_{d, \sigma}^{*} \mathcal{T}_{d, l}, \bar{p}_{d, \sigma}^{*} \dot{\mathcal{X}}_{\sigma}\right) \\
= & \pi_{*} \mathcal{E} x t^{1}\left(\bar{\phi}_{d, r, \sigma}^{*} \bar{a}_{d, \sigma}^{*} \bar{q}_{d, \sigma}^{*} \mathcal{T}_{d, l}, \bar{\phi}_{d, r, \sigma}^{*} \bar{a}_{d, \sigma}^{*} \bar{\rho}_{d, \sigma}^{*} \dot{\mathcal{X}}_{\sigma}\right) \\
= & \pi_{*} \mathcal{E} x t^{1}\left(\bar{f}^{*} \bar{\psi}_{d, \underline{r}, l}^{*} \mathcal{T}_{d, l}, \bar{g}^{*} \dot{\mathcal{X}}_{\sigma}\right)
\end{aligned}
$$


where

$$
f:=(1 \times \stackrel{\circ}{q}, \sigma)\left(1 \times a_{r, \sigma}\right), \quad g:=\stackrel{\circ}{p}_{d, \sigma} a_{d, \sigma} \phi_{d, r, \sigma} .
$$

Set $Q Q P:=Q_{d, r} \times_{Q_{r}} Q_{r, l} \times{ }_{Q_{l}} \stackrel{\circ}{P}_{\sigma}$. Since $R_{d, r, \sigma}$ is a projective bundle over $Q Q P$, the relative cotangent bundle $\Omega_{R_{d, r, \sigma} / Q Q P}$ fits into the following exact sequence

$0 \rightarrow \Omega_{R_{d, r, \sigma} / Q Q P} \rightarrow\left(\left(1 \times a_{r, \sigma}\right)^{*}\left(\psi_{\underline{d}, r, l} \times 1\right)^{*} \pi_{*} \mathcal{E} x t^{1}\left(\overline{\bar{q}}_{r, \sigma}^{*} \mathcal{T}_{r, l}, \overline{\bar{p}}_{r, \sigma}^{*} \dot{\mathcal{X}}_{\sigma}\right)\right)^{\vee}(-1) \rightarrow \mathcal{O}_{R_{d, r, \sigma}} \rightarrow 0$

We have

$$
\left(1 \times a_{r, \sigma}\right)^{*}\left(\psi_{\underline{d}, r, l} \times 1\right)^{*} \pi_{*} \mathcal{E} x t^{1}\left(\stackrel{\bar{q}}{q}_{r, \sigma}^{*} \mathcal{T}_{r, l}, \overline{\bar{p}}_{r, \sigma}^{*} \stackrel{\circ}{\mathcal{X}}_{\sigma}\right)=\pi_{*} \mathcal{E} x t^{1}\left(\bar{f}^{*} \bar{\psi}_{\underline{d}, r, l}^{*} \mathcal{T}_{r, l}, \bar{g}^{*} \stackrel{\circ}{\mathcal{X}}_{\sigma}\right)
$$

based on the equalities $\stackrel{\circ}{q}, \sigma_{r}\left(\psi_{\underline{d}, r, l} \times 1\right)\left(1 \times a_{r, \sigma}\right)=\psi_{\underline{d}, r, l} f$ and $\stackrel{\circ}{p}, \sigma_{r}\left(\psi_{\underline{d}, r, l} \times 1\right)(1 \times$ $\left.a_{r, \sigma}\right)=g$.

We make a diagram

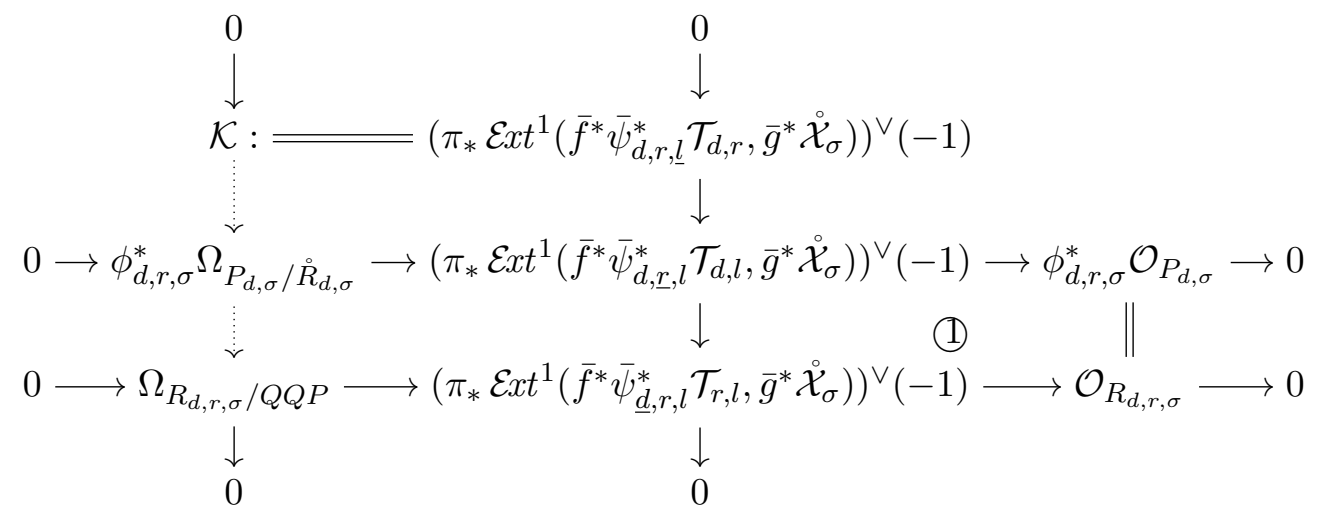

where

- the last row is the exact sequence (5.13),

- the middle row is the exact sequence (5.14), and

- the middle column is obtained by applying $\pi_{*} \mathcal{E} x t^{1}\left(\bar{f}^{*}(-), \bar{g}^{*} \dot{\mathcal{X}}_{\sigma}\right)$ to the exact sequence (5.4), then taking dual, and lastly twisting by $\mathcal{O}_{R_{d, r, \sigma}}(-1)$.

The commutativity of the rectangle (1) in the diagram (5.15) follows from diagram (5.6) and the identifications (5.8). Thus, we have induced maps (the dotted arrows) in the first column and the first column is exact. Restricting the first column to $\stackrel{\circ}{R}_{d, r, \sigma}$, we obtain an exact sequence

$$
0 \rightarrow \stackrel{\circ}{\mathcal{K}} \rightarrow \stackrel{\circ}{\phi}_{d, r, \sigma}^{*} \Omega_{P_{d, \sigma} / \stackrel{\circ}{R}, \sigma} \rightarrow \Omega_{\stackrel{\circ}{R}_{d, r, \sigma} / Q Q P} \rightarrow 0
$$


where $\stackrel{\circ}{\mathcal{K}}:=\left.\mathcal{K}\right|_{\stackrel{\circ}{R, r, \sigma}}$. We have

$$
\stackrel{\grave{\mathcal{K}}}{=}\left(\pi_{*} \mathcal{E} x t^{1}\left(\stackrel{\bar{f}}{*}^{*} \bar{\psi}_{d, r, \underline{l}}^{*} \mathcal{T}_{d, r}, \overline{\mathrm{g}}^{*} \dot{\mathcal{X}}_{\sigma}\right)\right)^{\vee}(-1)
$$

where $\stackrel{\circ}{f}$ and $\stackrel{\circ}{g}$ are the restrictions of $f$ and $g$ to $\stackrel{\circ}{R}_{d, r, \sigma}$.

Recall that $\psi_{d, r, l}: Q_{d, r, l} \rightarrow Q_{d, l}$ is a finite morphism. Since $\mathbb{k}$ is assumed to be of characteristic 0, the function field extension $K\left(Q_{d, r, l}\right) / K\left(Q_{d, l}\right)$ is separable. Therefore, we have an exact sequence of relative cotangent sheaves

$$
0 \rightarrow \psi_{d, \underline{r}, l}^{*} \Omega_{Q_{d, l}} \rightarrow \Omega_{Q_{d, r, l}} \rightarrow \Omega_{Q_{d, r, l} / Q_{d, l}} \rightarrow 0
$$

where both $\Omega_{Q_{d, l}}$ and $\Omega_{Q_{d, r, l}}$ are locally free of the same rank while $\Omega_{Q_{d, r, l} / Q_{d, l}}$ is torsion.

We form the following diagram

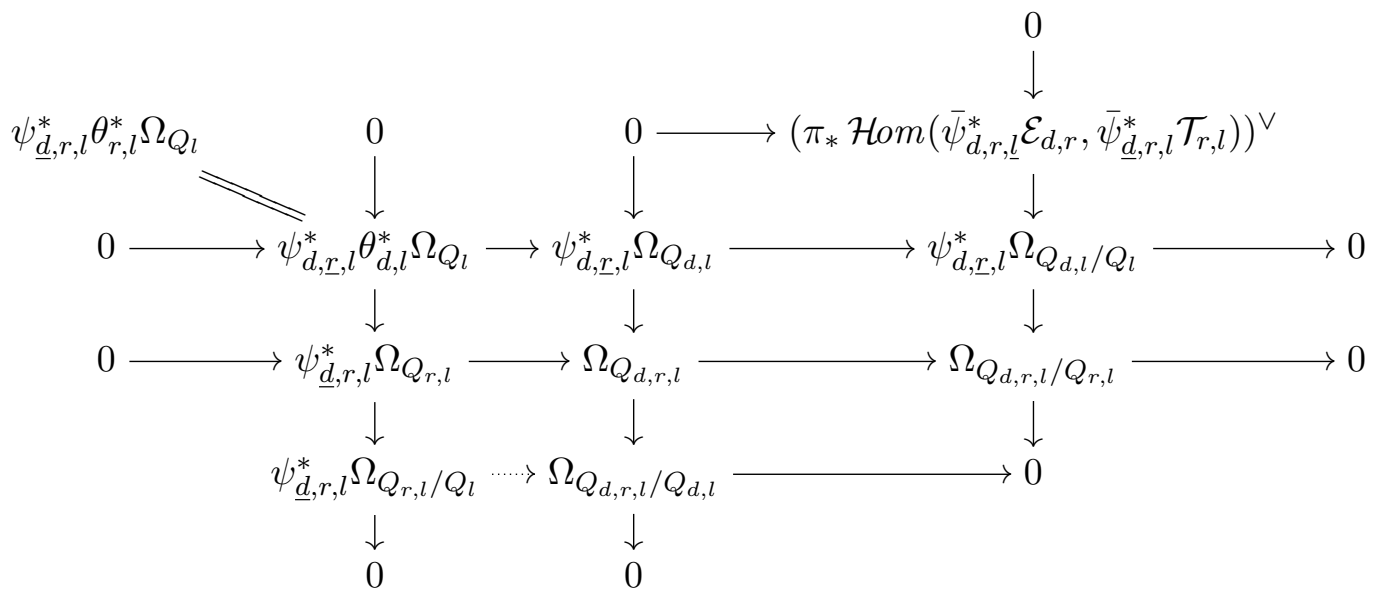

Applying the Snake Lemma to the third row and the fourth row of the above diagram, we obtain an exact sequence by connecting the second row with the last row:

$$
0 \rightarrow\left(\pi_{*} \mathcal{H o m}\left(\bar{\psi}_{d, r, \underline{l}}^{*} \mathcal{E}_{d, r}, \bar{\psi}_{\underline{d}, r, l}^{*} \mathcal{T}_{r, l}\right)\right)^{\vee} \rightarrow \psi_{\underline{d}, r, l}^{*} \Omega_{Q_{r, l} / Q_{l}} \rightarrow \Omega_{Q_{d, r, l} / Q_{d, l}} \rightarrow 0
$$


This sequence fits into the following commutative diagram

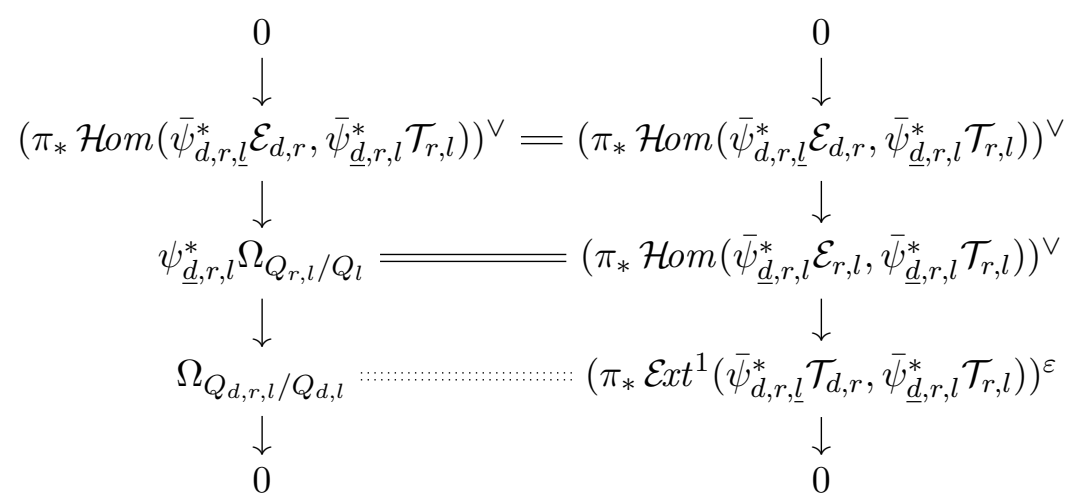

where the identification in the second row follows from the canonical identification $\mathcal{T}_{Q_{r, l} / Q_{l}}=\pi_{*} \mathcal{H} \operatorname{lom}\left(\bar{\psi}_{d, r, \underline{l}}^{*} \mathcal{E}_{d, r}, \bar{\psi}_{\underline{d}, r, l}^{*} \mathcal{T}_{r, l}\right)$. So we obtain an identification of the quotients

$$
\Omega_{Q_{d, r, l} / Q_{d, l}}=\left(\pi_{*} \mathcal{E} x t^{1}\left(\bar{\psi}_{d, r, \underline{l}}^{*} \mathcal{T}_{d, r}, \bar{\psi}_{\underline{d}, r, l}^{*} \mathcal{T}_{r, l}\right)\right)^{\varepsilon}
$$

Since $Q_{d, r, l}$ is smooth over $Q_{l}, Q Q P=Q_{d, r, l} \times{ }_{Q_{l}} \stackrel{\circ}{P}_{\sigma}$ is smooth over $\stackrel{\circ}{P}_{\sigma}$. The morphism $\psi_{d, \underline{r}, l} \times 1: Q Q P \rightarrow \stackrel{\circ}{R}_{d, \sigma}$ is obtained from $\psi_{d, \underline{r}, l}$ by the base change $\stackrel{\circ}{q}_{d, \sigma}: \stackrel{\circ}{R}_{d, \sigma} \rightarrow Q_{d, l}$, hence we have the following commutative diagram

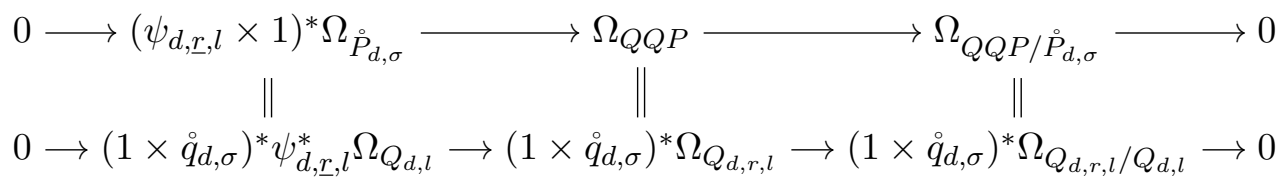


The conormal bundle $\mathcal{N}_{\mathscr{R}_{d, r, \sigma} / P_{d, \sigma}}^{\vee}$ fits into the following commutative diagram

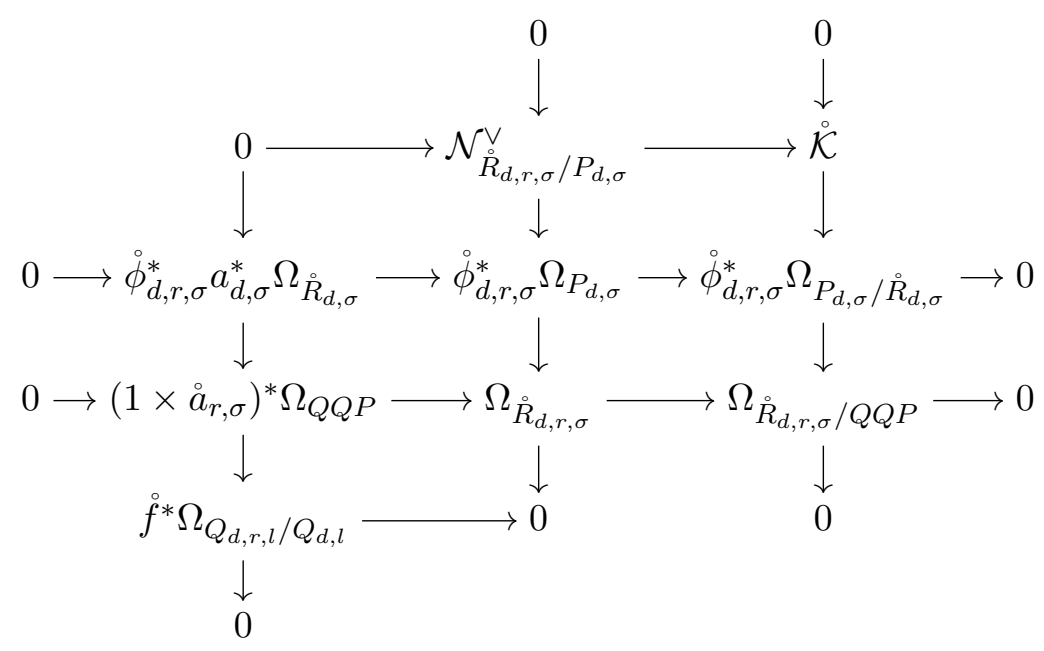

Applying the Snake Lemma to the third and fourth rows, we obtain an exact sequence by connecting the second row with the fifth row:

$$
0 \rightarrow \mathcal{N}_{\stackrel{R}{R}, r, \sigma^{\vee} / P_{d, \sigma}}^{\vee} \rightarrow \stackrel{\mathcal{K}}{\rightarrow} \rightarrow f^{*} \Omega_{Q_{d, r, l} / Q_{d, l}} \rightarrow 0
$$

The above sequence fits into the following commutative diagram

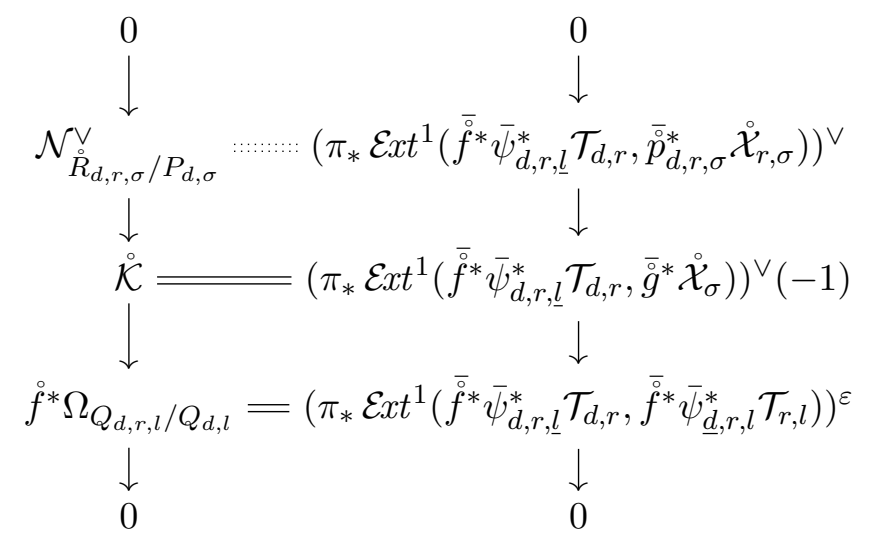

where the second column is obtained as follows: first we can rewrite the last row of diagram (5.6) as

$$
0 \rightarrow\left(\bar{g}^{*} \stackrel{\circ}{\sigma}_{\sigma}\right)(0,1) \rightarrow \bar{p}_{d, r, \sigma}^{*} \mathcal{X}_{r, \sigma} \rightarrow \bar{f}^{*} \bar{\psi}_{\underline{d}, r, l} \mathcal{T}_{r, l} \rightarrow 0
$$

next the restriction to $\mathbb{P}^{1} \times \stackrel{\circ}{R}_{d, r, \sigma}$ is

$$
0 \rightarrow\left(\overline{\bar{g}}^{*} \dot{\circ}_{\sigma}\right)(0,1) \rightarrow \overline{\bar{p}}_{d, r, \sigma}^{*} \dot{\mathcal{X}}_{r, \sigma} \rightarrow \overline{\bar{f}}^{*} \bar{\psi}_{\underline{d}, r, l} \mathcal{T}_{r, l} \rightarrow 0
$$


and next applying $\pi_{*} \mathcal{H o m}\left(\bar{\sigma}^{*} \bar{\psi}_{d, r, l}^{*} \mathcal{T}_{d, r},-\right)$ to the above sequence, and lastly taking dual, we obtain the second column in the diagram. Thus there is an induced identification as in the first row, which gives the natural identification

$$
\mathcal{N}_{\stackrel{\circ}{R}, r, \sigma / P_{d, \sigma}}=\pi_{*} \mathcal{E} x t^{1}\left(\stackrel{\bar{f}}{f}^{*} \bar{\psi}_{d, r, \underline{l}}^{*} \mathcal{T}_{d, r}, \overline{\bar{p}}_{d, r, \sigma}^{*} \stackrel{\circ}{r, \sigma}\right)=\pi_{*} \mathcal{E} x t^{1}\left(\overline{\bar{q}}_{d, r, \sigma}^{*} \mathcal{T}_{d, r}, \overline{\bar{p}}_{d, r, \sigma}^{*} \stackrel{\circ}{\mathcal{X}}_{r, \sigma}\right)
$$

\section{The MOdUlar InTERPRETATion}

The compactification $\widetilde{Q}_{d}$ is obtained by successively blowing up the Quot scheme $Q_{d}$ along $Z_{d, 0}, \cdots, Z_{d, d-1}$. We illustrate the process in the following diagram

$$
\begin{aligned}
& Z_{d, 0}^{d-1} \quad Z_{d, 1}^{d-1} \quad Z_{d, 2}^{d-1} \quad \cdots \quad Z_{d, d-1}^{d-1} \subset Q_{d}^{d-1}=\widetilde{Q}_{d} \\
& \downarrow \quad \downarrow \quad \downarrow \quad \downarrow \quad \downarrow \quad \downarrow \quad \text { along } Z_{d, d-1}^{d-2} \\
& \begin{array}{ccccccc}
\vdots & \vdots & \vdots & \vdots & \vdots & \vdots & \\
\downarrow & \downarrow & \downarrow & & \downarrow & \downarrow & \downarrow \\
Z_{d, 0}^{1} & Z_{d, 1}^{1} & Z_{d, 2}^{1} & \subset \cdots & \subset Z_{d, d-1}^{1} \subset & Q_{d}^{1} &
\end{array} \\
& \begin{array}{cccccc}
\downarrow & \downarrow & \downarrow & \downarrow & \downarrow & \downarrow \\
Z_{d, 0}^{0} & Z_{d, 1}^{0} \subset Z_{d, 2}^{0} \subset \cdots \subset Z_{d, d-1}^{0} \subset & Q_{d}^{0} &
\end{array} \\
& \downarrow \quad \downarrow \quad \downarrow \quad \downarrow \quad \downarrow \quad \leftarrow \text { along } Z_{d, 0} \\
& Z_{d, 0} \subset Z_{d, 1} \subset Z_{d, 2} \subset \cdots \subset Z_{d, d-1} \subset \quad Q_{d}
\end{aligned}
$$

Here, inductively, $Z_{d, 0}^{j}, \cdots, Z_{d, j}^{j}$ are the exceptional divisors created by the sequence of blowups $Q_{d}^{j} \rightarrow Q_{d}(0 \leq j \leq d-1)$; the nested subschemes $Z_{d, j+1}^{j} \subset$ $\cdots \subset Z_{d, d-1}^{j}$ are the proper transforms of the subschemes $Z_{d, j+1}^{j-1} \subset \cdots \subset Z_{d, d-1}^{j-1}$ (respectively); these are the subschemes lining up to be blown-up in the next steps. Below, we will provide modular meanings to the points of $Z_{d, 0}^{j}, \cdots, Z_{d, j}^{j}$ for all $j$. Thus every intermediate space $Q_{d}^{j}, j=0, \ldots, d-1$, also compactification of $\stackrel{Q}{d}_{d}$, admits parameter space interpretation (Proposition. 6.4). The case $Q_{d}^{d-1}$ is our final space $\widetilde{Q}_{d}$ (Corollary 6.5).

To proceed, we introduce the following

$$
\hat{Z}_{d, r}^{l}:=\left\{\begin{array}{ll}
\varnothing, & \text { if } r=l \\
Z_{d, r}^{l}, & \text { if } l<r<d
\end{array}, \quad \stackrel{Z}{d, r}^{l}:=Z_{d, r}^{l} \backslash \hat{Z}_{d, r-1}^{l}, \quad r=l+1, \ldots, d-1\right.
$$


Let $d>r \geq 0$. For each subsequence $\sigma \subset[r]:=(r, r-1, \cdots, 1,0) \in \Sigma$, we set

$$
E_{d, \sigma}^{r}:=\bigcap_{i \in \sigma} Z_{d, i}^{r} \backslash \bigcup_{i \in[r] \backslash \sigma} Z_{d, i}^{r}
$$

Then there is a stratification of $Q_{d}^{r}$ :

$$
Q_{d}^{r}=\left(Q_{d}^{r} \backslash \bigcup_{i \in[r]} Z_{d, i}^{r}\right) \sqcup \bigsqcup_{\sigma \subset[r]} E_{d, \sigma}^{r}=\left(Q_{d} \backslash Z_{d, r}\right) \sqcup \bigsqcup_{\sigma \subset[r]} E_{d, \sigma}^{r} .
$$

Lemma 6.1. Let $\tau \in \Sigma$, $t=\operatorname{lt}(\tau)$. We have

(1) $E_{d, \tau}^{j}=E_{d, \tau}^{m} \backslash Z_{d, j}^{m}$, for any $m$ and $j$ with $t \leq m<j<d$.

(2) if $l>t$ and $\sigma=(l, \tau)$, then $E_{d, \sigma}^{l}$ is the exceptional divisor of the blowup of $E_{d, \tau}^{t} \backslash \hat{Z}_{d, l-1}^{t}$ along $E_{d, \tau}^{t} \cap \stackrel{\circ}{d, l}_{d, l}$.

Proof. (1) Consider the composite blowup $b: Q_{d}^{j} \rightarrow Q_{d}^{m}, m<j$. We have $b^{-1}\left(Z_{d, i}^{m}\right)=Z_{d, i}^{j}$ for $i=0, \ldots, m$, and $b^{-1}\left(Z_{d, j}^{m}\right)=\bigcup_{i=m+1}^{j} Z_{d, i}^{j}$. Moreover, $b$ is an isomorphism away from $Z_{d, j}^{m}$. These facts give us identifications

$$
Q_{d}^{j} \backslash b^{-1}\left(Z_{d, j}^{m}\right)=Q_{d}^{m} \backslash Z_{d, j}^{m} \text {, and } Z_{d, i}^{j} \backslash b^{-1}\left(Z_{d, j}^{m}\right)=Z_{d, i}^{m} \backslash Z_{d, j}^{m} \text {, for } i=0, \ldots, m
$$

Therefore

$$
\begin{aligned}
E_{d, \tau}^{j} & =\bigcap_{i \in \tau} Z_{d, i}^{j} \backslash \bigcup_{i \in[j] \backslash \tau} Z_{d, i}^{j}=\left(\bigcap_{i \in \tau} Z_{d, i}^{j} \backslash \bigcup_{i \in[m] \backslash \tau} Z_{d, i}^{j}\right) \backslash \bigcup_{i=m+1}^{j} Z_{d, i}^{j} \\
& =\left(\bigcap_{i \in \tau} Z_{d, i}^{j} \backslash \bigcup_{i \in[m] \backslash \tau} Z_{d, i}^{j}\right) \backslash b^{-1}\left(Z_{d, j}^{m}\right) \\
& =\left(\bigcap_{i \in \tau} Z_{d, i}^{m} \backslash \bigcup_{i \in[m] \backslash \tau} Z_{d, i}^{m}\right) \backslash Z_{d, j}^{m}=E_{d, \tau}^{m} \backslash Z_{d, j}^{m}
\end{aligned}
$$

(2) By definition,

$$
E_{d, \sigma}^{l}=\bigcap_{i \in \sigma} Z_{d, i}^{l} \backslash \bigcup_{i \in[l] \backslash \sigma} Z_{d, i}^{l}=Z_{d, l}^{l} \cap \bigcap_{i \in \tau} Z_{d, i}^{l} \backslash \bigcup_{i \in[l] \backslash \sigma} Z_{d, i}^{l}
$$

We know $Z_{d, l}^{l}$ is the exceptional divisor of the blowup $Q_{d}^{l} \rightarrow Q_{d}^{l-1}$, which is along $Z_{d, l}^{l-1}$. Since $\left(\bigcap_{i \in \tau} Z_{d, i}^{l} \backslash \bigcup_{i \in[l] \backslash \sigma} Z_{d, i}^{l}\right) \subset Q_{d}^{l}$ is exactly the preimage of $\bigcap_{i \in \tau} Z_{d, i}^{l-1} \backslash \bigcup_{i \in[l] \backslash \sigma} Z_{d, i}^{l-1} \subset Q_{d}^{l-1}$ under the blowup, we have that $E_{d, \sigma}^{l}$ is the 
exceptional divisor of the (induced) blowup of $\bigcap_{i \in \tau} Z_{d, i}^{l-1} \backslash \bigcup_{i \in[l] \backslash \sigma} Z_{d, i}^{l-1}$ along $Z_{d, l}^{l-1} \cap \bigcap_{i \in \tau} Z_{d, i}^{l-1} \backslash \bigcup_{i \in[l] \backslash \sigma} Z_{d, i}^{l-1}$. Note that

$\bigcap_{i \in \tau} Z_{d, i}^{l-1} \backslash \bigcup_{i \in[l] \backslash \sigma} Z_{d, i}^{l-1}=\bigcap_{i \in \tau} Z_{d, i}^{l-1} \backslash\left(\bigcup_{i \in[t] \backslash \tau} Z_{d, i}^{l-1} \cup \bigcup_{i=t+1}^{l-1} Z_{d, i}^{l-1}\right)=E_{d, \tau}^{l-1} \backslash \bigcup_{i=t+1}^{l-1} Z_{d, i}^{l-1}$

If $l-1=t$, then $E_{d, \sigma}^{l}$ is the exceptional divisor of the blowup of $E_{d, \tau}^{t}$ along $Z_{d, t+1}^{t}$, and we are done. Now suppose $l-1>t$. Then under the identification $Q_{d}^{l-1} \backslash \bigcup_{i=t+1}^{l-1} Z_{d, i}^{l-1}=Q_{d}^{t} \backslash Z_{d, l-1}^{t}$, we have identifications $E_{d, \tau}^{l-1} \backslash \bigcup_{i=t+1}^{l-1} Z_{d, i}^{l-1}=$ $E_{d, \tau}^{t} \backslash Z_{d, l-1}^{t}$ and

$$
Z_{d, l}^{l-1} \backslash \bigcup_{i=t+1}^{l-1} Z_{d, i}^{l-1}=Z_{d, l}^{t} \backslash Z_{d, l-1}^{t}=\check{Z}_{d, l}^{t}
$$

Proposition 6.2. There is a collection of isomorphisms

$$
i_{d, \sigma}: P_{d, \sigma} \stackrel{\sim}{\rightarrow} E_{d, \sigma}^{l}
$$

one for each $\sigma \in \Sigma$ with $l:=\operatorname{lt}(\sigma)<d$, such that the following properties hold:

(1) $i_{d, \sigma}$ maps $Y_{d, r, \sigma}$ onto $E_{d, \sigma}^{l} \cap Z_{d, r}^{l}$ for all $r, l<r<d ; ;$

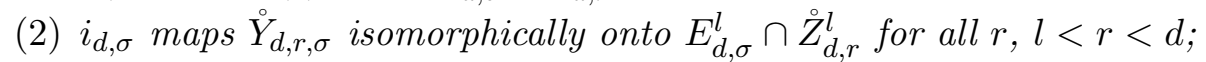

(3) The following diagram commutes:

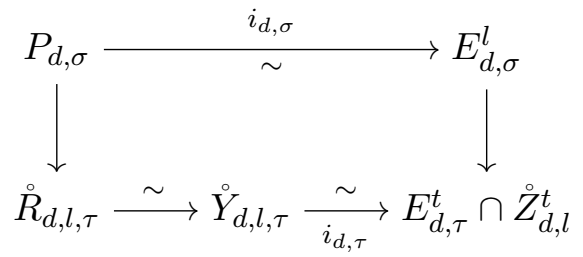

where $\sigma=(l, \tau)$ and $t=\operatorname{lt}(\tau)$;

(4) Let $e_{d, \sigma}: P_{d, \sigma} \hookrightarrow Q_{d}^{l}$ be the embedding obtained through the composition $P_{d, \sigma} \stackrel{\sim}{\rightarrow} E_{d, \sigma}^{l} \hookrightarrow Q_{d}^{l}$. Then the following diagram commutes

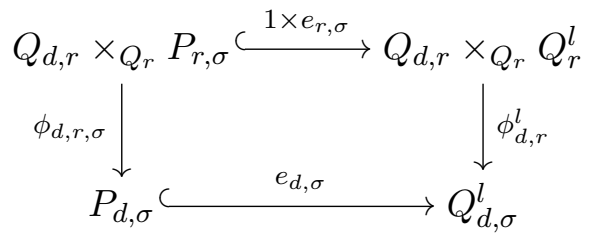

for all $r, l<r<d$. 
Proof. We prove by constructing the isomorphisms $i_{d, \sigma}$ explicitly, and this is done by induction on the length of $\sigma$. We first deal with the base case: $\sigma \in \Sigma_{1}$ or $\sigma=(l)$. In this case, we construct isomorphisms

$$
P_{d, l} \stackrel{\sim}{\rightarrow} E_{d, l}^{l}=Z_{d, l}^{l} \backslash \bigcup_{0 \leq i \leq l-1} Z_{d, i}^{l}
$$

which map $Y_{d, r, l}$ onto $E_{d, l}^{l} \cap Z_{d, r}^{l}$ and map $\stackrel{\circ}{Y}_{d, r, l}$ isomorphically onto $E_{d, l}^{l} \cap \stackrel{\circ}{Z}_{d, r}^{l}$ for all $d, r$ and $l$ such that $l<r<d$.

Recall that $Z_{d, l}^{l}$ is the exceptional divisor in the blowup $b: Q_{d}^{l} \rightarrow Q_{d}^{l-1}$ along $Z_{d, l}^{l-1}$. We have $b^{-1}\left(Z_{d, i}^{l-1}\right)=Z_{d, i}^{l}$ for $i=0, \ldots, l-1$, hence

$$
b: Q_{d}^{l} \backslash \bigcup_{i=0}^{l-1} Z_{d, i}^{l} \rightarrow Q_{d}^{l-1} \backslash \bigcup_{i=0}^{l-1} Z_{d, i}^{l-1}
$$

is the blowup along $Z_{d, l}^{l-1} \backslash \bigcup_{i=0}^{l-1} Z_{d, i}^{l-1}$ with exceptional divisor $Z_{d, l}^{l} \backslash \bigcup_{i=0}^{l-1} Z_{d, i}^{l}=$ $E_{d, l}^{l}$. Note that $Q_{d}^{l-1} \backslash \bigcup_{i=0}^{l-1} Z_{d, i}^{l-1}=Q_{d} \backslash Z_{d, l-1}$ and $Z_{d, l}^{l-1} \backslash \bigcup_{i=0}^{l-1} Z_{d, i}^{l-1}=Z_{d, l} \backslash$ $Z_{d, l-1}=\stackrel{\circ}{Z}_{d, l}$. Therefore $E_{d, l}^{l}$ is the projective bundle $\mathbb{P}\left(\mathcal{N}_{\dot{Z}_{d, l} / Q_{d}}\right)$ over $\stackrel{\circ}{Z}_{d, l}$. Since $\stackrel{\circ}{Z}_{d, l} \simeq \stackrel{\circ}{Q}_{d, l}$ and by Proposition 4.2

$$
\mathcal{N}_{\stackrel{Q}{d, l} / Q_{d}}=\left.\pi_{*} \mathcal{E} x t^{1}\left(\mathcal{T}_{d, l}, \bar{\theta}_{d, l}^{*} \mathcal{F}_{l}\right)\right|_{\stackrel{Q}{d, l}_{l}}=\pi_{*} \mathcal{E} x t^{1}\left(\bar{q}_{d, l}^{*} \mathcal{T}_{d, l}, \bar{p}_{d, l}^{*} \stackrel{\circ}{\mathcal{X}}_{l}\right)
$$

we know $E_{d, l}^{l}$ is isomorphic to the projective bundle

$$
P_{d, l}=\mathbb{P}\left(\pi_{*} \mathcal{E} x t^{1}\left(\overline{\bar{q}}_{d, l}^{*} \mathcal{T}_{d, l}, \overline{\bar{p}}_{d, l}^{*} \stackrel{\circ}{\mathcal{X}}_{l}\right)\right)
$$

over $\stackrel{\circ}{d, l}_{l}$. So we obtain an embedding $e_{d, l}: P_{d, l} \hookrightarrow Q_{d}^{l}$ through the composition

$$
P_{d, l} \stackrel{\sim}{\rightarrow} E_{d, l}^{l} \hookrightarrow Q_{d}^{l}
$$

For each $r$ with $l<r<d$, we have a commtative diagram

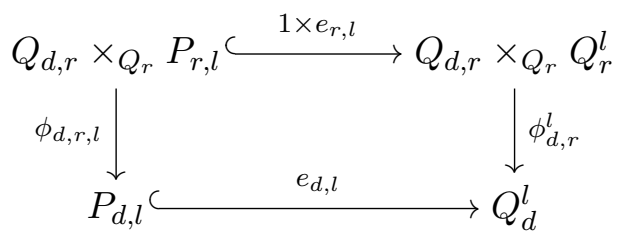

We have that $\operatorname{Im}\left(\phi_{d, r, l}\right)=Y_{d, r, l}$, that $\operatorname{Im}\left(1 \times e_{r, l}\right)=Q_{d, r} \times{ }_{Q_{r}} E_{r, l}^{l}$, and that $\phi_{d, r}^{l}$ maps $Q_{d, r} \times_{Q_{r}} E_{r, l}^{l}$ onto $E_{d, l}^{l} \cap Z_{d, r}^{l}$. It follows that $e_{d, l}$ maps $Y_{d, r, l}$ onto $E_{d, l}^{l} \cap Z_{d, r}^{l}$. 
Next, we show that $e_{d, l}: P_{d, l} \rightarrow Q_{d}^{l}$ maps $\stackrel{\circ}{Y}_{d, r, l}$ isomorphically onto $E_{d, l}^{l} \cap \stackrel{\circ}{Z}_{d, r}^{l}$ for $l<r<d$. We have a commutative diagram

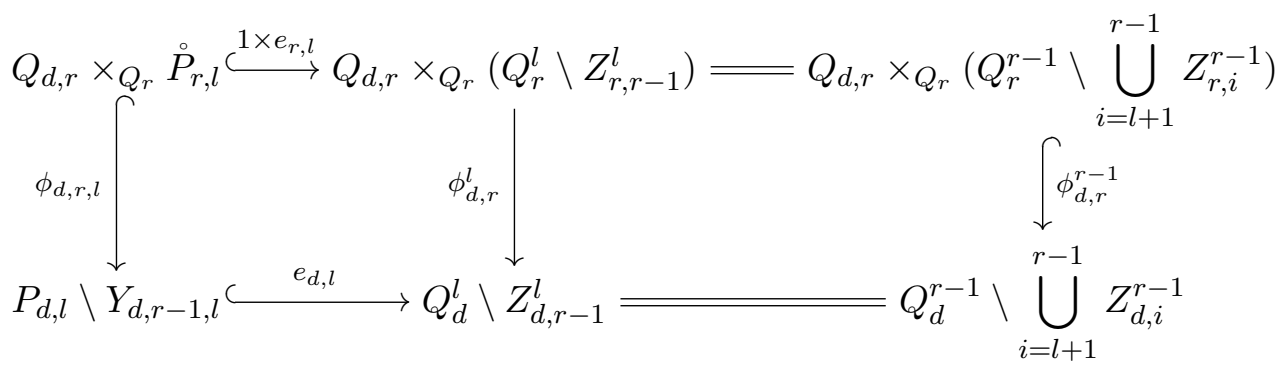

We see that $e_{r, l}$ maps $\stackrel{\circ}{P}_{r, l}$ isomorphically onto $E_{r, l}^{l} \backslash Z_{r, r-1}^{l}$ in $Q_{r}^{l} \backslash Z_{r, r-1}^{l}, E_{r, l}^{l} \backslash$ $Z_{r, r-1}^{l}$, which can be identified with $E_{r, l}^{r-1} \subset Q_{r}^{r-1} \backslash \bigcup_{i=l+1}^{r-1} Z_{r, i}^{r-1}$ (by Lemma 6.1), and $\phi_{d, r}^{r-1}$ maps $Q_{d, r} \times{ }_{Q_{r}} E_{r, l}^{r-1}$ isomorphically onto $E_{d, l}^{r-1} \cap Z_{d, r}^{r-1}$, which is identified with $E_{d, l}^{l} \cap \stackrel{\circ}{Z}_{d, r}^{l}$. On the other hand, $\phi_{d, r, l}$ maps $Q_{d, r} \times{ }_{Q_{r}} \stackrel{\circ}{P}, l^{\prime}$ isomorphically onto $\stackrel{\circ}{Y}_{d, r, l} \subset P_{d, l} \backslash Y_{d, r-1, l}$. It follows that $e_{d, l}$ maps $\stackrel{\circ}{Y}_{d, r, l}$ isomorphically onto $E_{d, l}^{l} \cap \stackrel{\circ}{Z}_{d, r}^{l}$. Thus the case that $\sigma \in \Sigma_{1}$ is constructed.

Suppose we have constructed the isomorphisms for all $\sigma \in \Sigma_{m}$ for some $m$. We now construct the isomorphisms for $\sigma \in \Sigma_{m+1}$. Now write $\sigma=(l, \tau)$ and let $t=\operatorname{lt}(\tau)$. By induction hypothesis, for any $d>t$, we have an isomorphism $P_{d, \tau} \stackrel{\sim}{\rightarrow} E_{d, \tau}^{t}$ which maps $Y_{d, r, \tau}$ onto $E_{d, \tau}^{t} \cap Z_{d, r}^{t}$ and maps $\stackrel{\circ}{d, r, \tau}_{\text {isomorphically }}$ onto $E_{d, \tau}^{t} \cap \stackrel{o}{Z}_{d, r}^{t}$. Let $d>l$. By Lemma $6.1, E_{d, \sigma}^{l}$ is the exceptional divisor of the blowup of $E_{d, \tau}^{t} \backslash \tilde{Z}_{d, l-1}^{t}$ along $E_{d, \tau}^{t} \cap \stackrel{\circ}{Z}_{d, l}^{t}$. Since the isomorphism $P_{d, \tau} \stackrel{\sim}{\rightarrow} E_{d, \tau}^{t}$ maps $\stackrel{\circ}{Y}_{d, l, \tau}$ isomorphically onto $E_{d, \tau}^{t} \cap \stackrel{\complement}{Z}_{d, l}^{t}$, we have an isomorphism

$$
\mathrm{Bl}_{\stackrel{Y}{Y}, l, \tau}\left(P_{d, \tau} \backslash Y_{d, l-1, \tau}\right) \stackrel{\sim}{\rightarrow} \mathrm{Bl}_{E_{d, \tau}^{t} \cap \check{Z}_{d, l}^{t}}\left(E_{d, \tau}^{t} \backslash \tilde{Z}_{d, l-1}^{t}\right)
$$

which maps the exceptional divisor $E_{d, \sigma}^{l}$ of the blowup $\mathrm{Bl}_{\dot{Y}_{d, l, \tau}}\left(P_{d, \tau} \backslash Y_{d, l-1, \tau}\right)$ isomorphically onto the exceptional divisor $E_{d, \sigma}^{l}$ of $\mathrm{Bl}_{E_{d, \tau}^{t} \cap \check{Z}_{d, l}^{t}}\left(E_{d, \tau}^{t} \backslash Z_{d, l-1}^{t}\right)$. On the other hand, the exceptional divisor of $\mathrm{Bl}_{\dot{Y}_{d, l, \tau}}\left(P_{d, \tau} \backslash Y_{d, l-1, \tau}\right)$ is isomorphic to the projective normal bundle of $\stackrel{\circ}{d, l, \tau}_{\text {in }} P_{d, \tau} \backslash Y_{d, l-1, \tau}$ or just in $P_{d, \tau}$. We know that $\phi_{d, l, \tau}: Q_{d, l} \times{ }_{Q_{l}} P_{l, \tau} \rightarrow P_{d, \tau}$ maps $Q_{d, l} \times{ }_{Q_{l}} \stackrel{\circ}{P}_{l, \tau}$ isomorphically onto $\stackrel{\circ}{Y}_{d, l, \tau}$, and by Proposition 5.6 the normal bundle of $Q_{d, l} \times{ }_{Q_{l}} \stackrel{\circ}{P}_{l, \tau}$ in $P_{d, \tau}$ is $\pi_{*} \mathcal{E} x t^{1}\left(\overline{\bar{q}}_{d, l, \tau}^{*} \mathcal{T}_{d, l}, \overline{\bar{p}}_{d, l, \tau}^{*} \dot{\mathcal{X}}_{l, \tau}\right)$. Hence the projective normal bundle of $\dot{Y}_{d, l, \tau}$ in $P_{d, \tau}$ is isomorphic to $\mathbb{P}\left(\pi_{*} \mathcal{E} x t^{1}\left(\overline{\stackrel{\rho}{q}}_{d, l, \tau}^{*} \mathcal{T}_{d, l}, \overline{\dot{p}}_{d, l, \tau}^{*} \dot{\mathcal{X}}_{l, \tau}\right)\right)=P_{d, l, \tau}=P_{d, \sigma}$. Thus we obtain an isomorphism $P_{d, \sigma} \stackrel{\sim}{\rightarrow} E_{d, \sigma}^{l}$. Next we show that this isomorphism maps $Y_{d, r, \sigma}$ onto $E_{d, \sigma}^{l} \cap Z_{d, r}^{l}$ and maps $\stackrel{\circ}{Y}_{d, r, \sigma}$ isomorphically onto $E_{d, \sigma} \cap \stackrel{\circ}{Z}_{d, r}^{l}$. 
Let $e_{d, \sigma}: P_{d, \sigma} \hookrightarrow Q_{d}^{l}$ denote the embedding obtained from the composition $P_{d, \sigma} \stackrel{\sim}{\rightarrow} E_{d, \sigma}^{l} \hookrightarrow Q_{d, \sigma}^{l}$ for each $d>l$. Let $d>r>l$. Then we have a commutative diagram

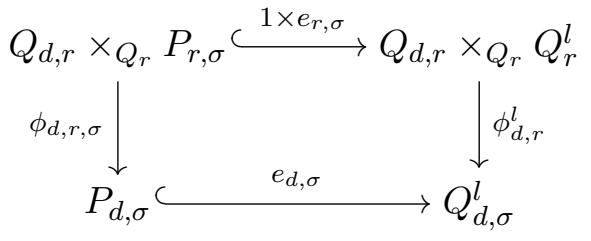

We have that $\operatorname{Im}\left(\phi_{d, r, \sigma}\right)=Y_{d, r, \sigma}$, that $\operatorname{Im}\left(1 \times e_{r, \sigma}\right)=Q_{d, r} \times{ }_{Q_{r}} E_{r, \sigma}^{l}$, and that $\phi_{d, r}^{l} \operatorname{maps} Q_{d, r} \times{ }_{Q_{r}} E_{r, \sigma}^{l}$ onto $E_{d, \sigma}^{l} \cap Z_{d, r}^{l}$. It follows that $e_{d, \sigma}$ maps $Y_{d, r, \sigma}$ onto $E_{d, \sigma}^{l} \cap Z_{d, r}^{l}$.

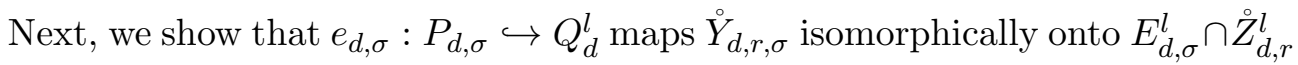
for any $r, l<r<d$. We have a commutative diagram

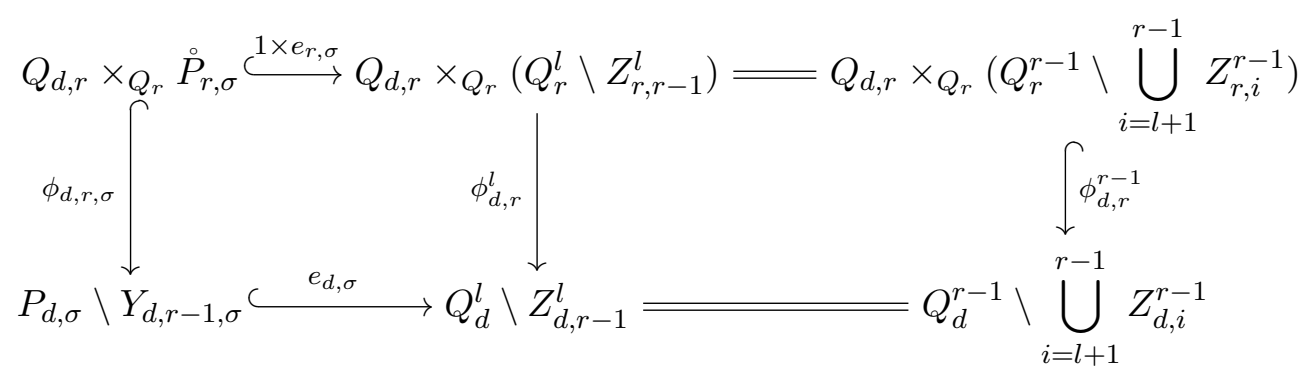

We see that $e_{r, \sigma}$ maps $\stackrel{\circ}{P}_{r, \sigma}$ isomorphically onto $E_{r, \sigma}^{l} \backslash Z_{r, r-1}^{l}$ in $Q_{r}^{l} \backslash Z_{r, r-1}^{l}$, that $E_{r, \sigma}^{l} \backslash Z_{r, r-1}^{l}$ can be identified with $E_{r, \sigma}^{r-1} \subset Q_{r}^{r-1} \backslash \bigcup_{i=l+1}^{r-1} Z_{r, i}^{r-1}$ (by Lemma 6.1), and that $\phi_{d, r}^{r-1}$ maps $Q_{d, r} \times{ }_{Q_{r}} E_{r, l}^{r-1}$ isomorphically onto $E_{d, l}^{r-1} \cap Z_{d, r}^{r-1}$, which is identified with $E_{d, l}^{l} \cap \stackrel{\circ}{Z}_{d, r}^{l}$. On the other hand, $\phi_{d, r, \sigma}$ maps $Q_{d, r} \times{ }_{Q_{r}} \stackrel{\circ}{P}, \sigma^{\prime}$ in isomorphically onto $\stackrel{\circ}{Y}_{d, r, \sigma} \subset P_{d, \sigma} \backslash Y_{d, r-1, \sigma}$. It follows that $e_{d, \sigma}$ maps $\stackrel{\circ}{Y}_{d, r, \sigma}$ isomorphically onto $E_{d, \sigma}^{l} \cap \stackrel{\circ}{Z}_{d, r}^{l}$. Thus the case that $\sigma \in \Sigma_{m+1}$ is constructed.

Proposition 6.3. Let $\sigma \in \Sigma$. Then for any $d$ and $l, d>l \geq \operatorname{lt}(\sigma)$, there is an

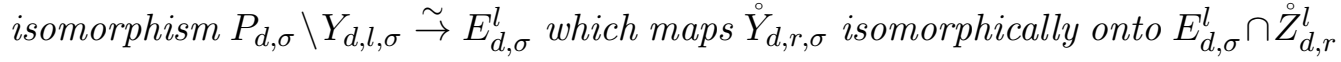
for any $r, l<r<d$;

Proof. The case that $l=\operatorname{lt}(\sigma)$ is proved in the above proposition. We now prove the case that $l>\operatorname{lt}(\sigma)$. Let $t=\operatorname{lt}(\sigma)$. Then by the above proposition, we have an isomorphism $P_{d, \sigma} \stackrel{\sim}{\rightarrow} E_{d, \sigma}^{t}$ which maps $Y_{d, l, \sigma}$ onto $E_{d, \sigma}^{t} \cap Z_{d, l}^{t}$ for each $r$. This isomorphism restricts to an isomorphism $P_{d, \sigma} \backslash Y_{d, l, \sigma} \stackrel{\sim}{\rightarrow} E_{d, \sigma}^{t} \backslash Z_{d, l}^{t}$. Under the 
identification $Q_{d}^{t} \backslash Z_{d, l}^{t}=Q_{d}^{l} \backslash \bigcup_{i=t+1}^{l} Z_{d, i}^{l}, E_{d, \sigma}^{t} \backslash Z_{d, l}^{t}$ is identified with $E_{d, \sigma}^{l}$, and $E_{d, \sigma}^{t} \cap \stackrel{\circ}{Z}_{d, r}^{t}$ is identified with $E_{d, \sigma}^{l} \cap \stackrel{\Xi}{Z}_{d, r}^{l}$ for any $r, l<r<d$. Hence we obtain an isomorphism $P_{d, \sigma} \backslash Y_{d, l, \sigma} \stackrel{\sim}{\rightarrow} E_{d, \sigma}^{l}$ which maps $\stackrel{\circ}{Y}_{d, r, \sigma}$ isomorphically onto $E_{d, \sigma}^{l} \cap \stackrel{\circ}{Z}_{d, r}^{l}$ for any $r, l<r<d$.

Let $\sigma=\left(l_{m}, \cdots, l_{1}\right) \in \Sigma$ and $d>l_{m}$. We have the following commutative diagram

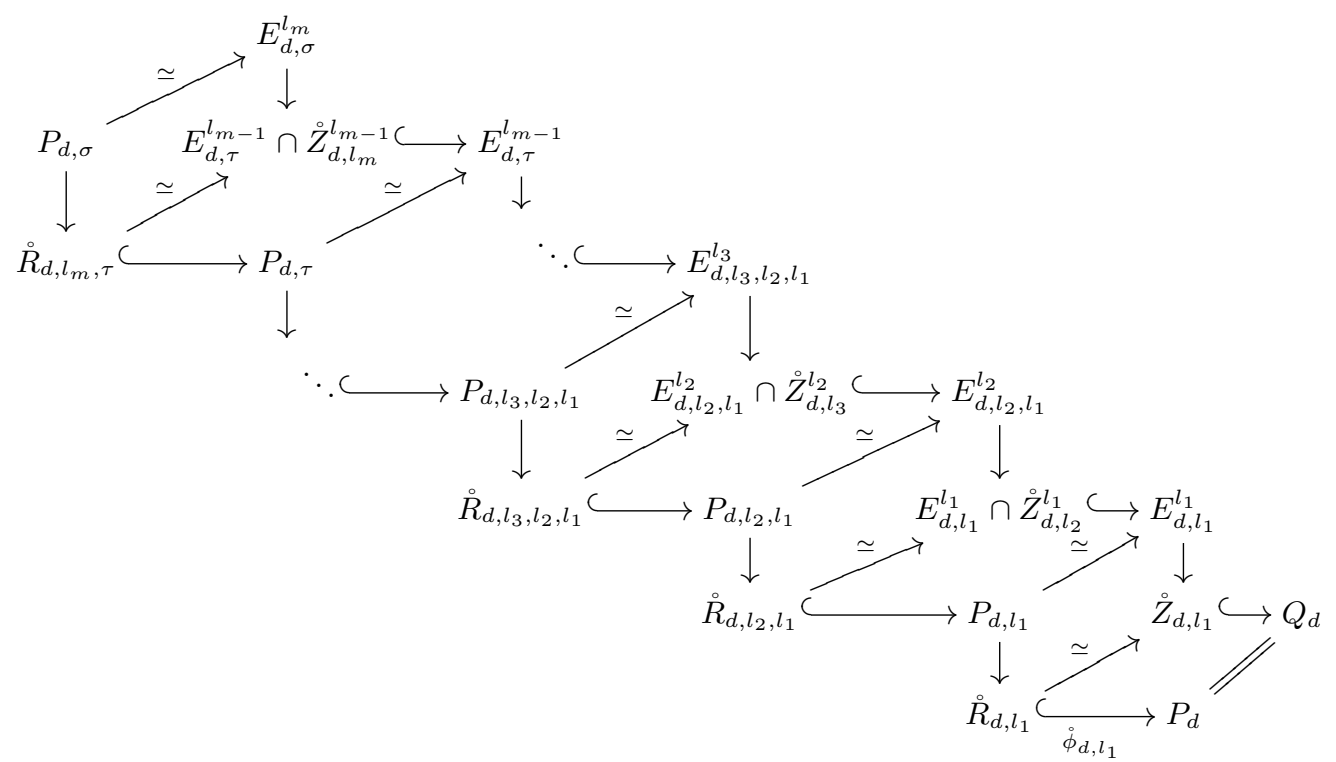

where $\tau=\left(l_{m-1}, \cdots, l_{1}, l_{0}\right)$. Thus we obtain a sequence of canonical identifications:

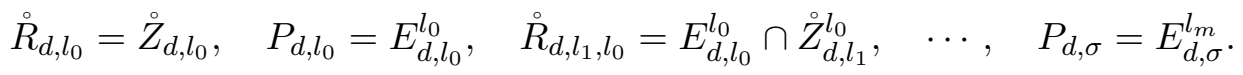

Recall that $Q_{d}$ parametrizes quotient of the form $V_{\mathbb{P} 1} \rightarrow X_{1}$ with $\operatorname{deg} X_{1}=d$, and the subset $\stackrel{\circ}{Z}_{d, l_{1}}$ of $Q_{d}$ parametrizes such quotients with $\operatorname{deg} X_{1}^{\mathbf{t}}=d-l_{1}$.

Let $x_{1}=\left[V_{\mathbb{P}^{1}} \rightarrow X_{1}\right] \in \stackrel{\circ}{Z}_{d, l_{1}}=\stackrel{\circ}{R}_{d, l_{1}}$. Using the identification,

$$
E_{d, l_{1}}^{l_{1}}=P_{d, l_{1}}=\mathbb{P}\left(\pi_{*} \mathcal{E} x t^{1}\left(\overline{\bar{q}}_{d, l_{1}}^{*} \mathcal{T}_{d, l_{1}}, \bar{p}_{d, l_{1}}^{*} \dot{\mathcal{X}}_{l_{1}}\right)\right),
$$

we see that the fiber of $E_{d, l_{1}}^{l_{1}} \rightarrow \stackrel{\circ}{Z}_{d, l_{1}}$ over $x_{1}$ is $\mathbb{P}\left(\operatorname{Ext}^{1}\left(\left(\overline{\bar{q}}_{d, l}^{*} \mathcal{T}_{d, l}\right)_{x_{1}},\left(\overline{\bar{p}}_{d, l}^{*} \dot{\mathcal{X}}_{l}\right)_{x_{1}}\right)\right)$. On the other hand, we have an exact sequence

$$
0 \rightarrow \overline{\bar{q}}_{d, l_{1}}^{*} \mathcal{T}_{d, l_{1}} \rightarrow \overline{\bar{\phi}}_{d, l_{1}}^{*} \mathcal{X}_{d} \rightarrow \overline{\bar{p}}_{d, l_{1}}^{*}{\stackrel{\circ}{l_{1}}} \rightarrow 0
$$


whose restriction to $\mathbb{P}^{1} \times\left\{x_{1}\right\}$,

$$
0 \rightarrow\left(\overline{\bar{q}}_{d, l_{1}}^{*} \mathcal{T}_{d, l_{1}}\right)_{x_{1}} \rightarrow\left(\bar{\phi}_{d, l_{1}}^{*} \mathcal{X}_{d}\right)_{x_{1}} \rightarrow\left(\overline{\bar{p}}_{d, l_{1}}^{*} \dot{\mathcal{X}}_{l_{1}}\right)_{x_{1}} \rightarrow 0
$$

is also an exact sequence. Since $\dot{\phi}_{d, l_{1}}$ is an inclusion map, we have that $\left(\bar{\phi}_{d, l_{1}}^{*} \mathcal{X}_{d}\right)_{x_{1}}=$ $X_{1}$. Hence $\left(\overline{\hat{q}}_{d, l_{1}}^{*} \mathcal{T}_{d, l_{1}}\right)_{x_{1}}=X_{1}^{\mathbf{t}}$ and $\left(\bar{p}_{d, l_{1}}^{*} \dot{\mathcal{X}}_{l_{1}}\right)_{x_{1}}=X_{1}^{\mathbf{f}}$, and the fiber over $x_{1}$ is $\mathbb{P}\left(\operatorname{Ext}^{1}\left(X_{1}^{\mathbf{t}}, X_{1}^{\mathbf{f}}\right)\right)$, which parametrizes non-split extensions of the form $\left[X_{1}^{\mathbf{f}} \longmapsto\right.$ $\left.X_{2} \rightarrow X_{1}^{\mathbf{t}}\right]$. Thus $E_{d, l_{1}}^{l_{1}}$ parametrizes sequences of the form

$$
\left(\left[V_{\mathbb{P} 1} \rightarrow X_{1}\right],\left[X_{1}^{\mathbf{f}} \longmapsto X_{2} \rightarrow X_{1}^{\mathbf{t}}\right]\right) .
$$

with $\operatorname{deg} X_{1}^{\mathbf{t}}=d-l_{1}$ and $\operatorname{deg} X_{2}^{\mathbf{t}}<d-l_{1}$. Using the identification

$$
\stackrel{\circ}{R}_{d, l_{2}, l_{1}}=\stackrel{\circ}{Y}_{d, l_{2}, l_{1}}=E_{d, l_{1}}^{l_{1}} \cap \stackrel{\circ}{Z}_{d, l_{2}}^{l_{1}},
$$

we see that $E_{d, l_{1}}^{l_{1}} \cap \stackrel{\circ}{Z}_{d, l_{2}}^{l_{1}}$ parametrizes such sequences with $\operatorname{deg} X_{1}^{\mathbf{t}}=d-l_{1}$ and $\operatorname{deg} X_{2}^{\mathbf{t}}=d-l_{2}$, by the definition of $\stackrel{\circ}{Y}_{d, l_{2}, l_{1}}$.

Let $x_{2}=\left(\left[V_{\mathbb{P}^{1}} \rightarrow X_{1}\right],\left[X_{1}^{\mathbf{f}} \longrightarrow X_{2} \rightarrow X_{1}^{\mathbf{t}}\right]\right) \in E_{d, l_{1}}^{l_{1}} \cap \stackrel{\circ}{Z}_{d, l_{2}}^{l_{1}}=\stackrel{\circ}{R}_{d, l_{2}, l_{1}}$. Using the identification $E_{d, l_{2}, l_{1}}^{l_{2}}=P_{d, l_{2}, l_{1}}=\mathbb{P}\left(\mathcal{E} x t^{1}\left(\overline{\bar{q}}_{d, l_{2}, l_{1}}^{*} \mathcal{T}_{d, l_{2}}, \bar{p}_{d, l_{2}, l_{1}}^{*} \dot{\mathcal{X}}_{l_{2}, l_{1}}\right)\right)$, we have that the fiber of $E_{d, l_{2}, l_{1}}^{l_{2}} \rightarrow E_{d, l_{1}}^{l_{1}} \cap{\stackrel{\circ}{d, l_{2}}}_{l_{1}}^{l_{1}}$ over the point $x_{2}$ is

$$
\mathbb{P}\left(\mathcal{E} x t^{1}\left(\left(\overline{\stackrel{q}{q}}_{d, l_{2}, l_{1}}^{*} \mathcal{T}_{d, l_{2}}\right)_{x_{2}},\left(\overline{\bar{p}}_{d, l_{2}, l_{1}}^{*} \mathcal{X}_{l_{2}, l_{1}}\right)_{x_{2}}\right)\right) .
$$

On the other hand, we have an exact sequence

$$
0 \rightarrow \bar{q}_{d, l_{2}, l_{1}}^{*} \mathcal{T}_{d, l_{2}} \rightarrow \overline{\dot{\phi}}_{d, l_{2}, l_{1}}^{*} \mathcal{X}_{d, l_{1}} \rightarrow \overline{\bar{p}}_{d, l_{2}, l_{1}}^{*} \dot{\mathcal{X}}_{l_{2}, l_{1}} \rightarrow 0
$$

whose restriction to $\mathbb{P}^{1} \times\left\{x_{2}\right\}$,

$$
0 \rightarrow\left(\overline{\bar{q}}_{d, l_{2}, l_{1}}^{*} \mathcal{T}_{d, l_{2}}\right)_{x_{2}} \rightarrow\left(\stackrel{\bar{\rho}}{\phi}_{d, l_{2}, l_{1}}^{*} \mathcal{X}_{d, l_{1}}\right)_{x_{2}} \rightarrow\left(\overline{\bar{p}}_{d, l_{2}, l_{1}}^{*}{\stackrel{\circ}{\mathcal{X}_{2}, l_{1}}}_{x_{2}} \rightarrow 0\right.
$$

is also an exact sequence. Since $\bar{\phi}_{d, l_{2}, l_{1}}^{*}$ is an inclusion map, we have

$$
\left(\bar{\phi}_{d, l_{2}, l_{1}}^{*} \mathcal{X}_{d, l_{1}}\right)_{x_{2}}=X_{2} .
$$

Hence $\left(\overline{\bar{q}}_{d, l_{2}, l_{1}}^{*} \mathcal{T}_{d, l_{2}}\right)_{x_{2}}=X_{2}^{\mathbf{t}},\left(\overline{\bar{p}}_{d, l_{2}, l_{1}}^{*} \dot{\mathcal{X}}_{l_{2}, l_{1}}\right)_{x_{2}}=X_{2}^{\mathbf{f}}$, and the fiber over $x_{2}$ is $\mathbb{P}\left(\operatorname{Ext}^{1}\left(X_{2}^{\mathbf{t}}, X_{2}^{\mathbf{f}}\right)\right)$, which parametrizes non-split extensions of the form $\left[X_{2}^{\mathbf{f}} \longmapsto\right.$ $\left.X_{3} \rightarrow X_{2}^{\mathrm{t}}\right]$. Thus $E_{d, l_{2}, l_{1}}^{l_{2}}$ parametrizes sequences of the form:

$$
\left(\left[V_{\mathbb{P}^{1}} \rightarrow X_{1}\right],\left[X_{1}^{\mathbf{f}} \longmapsto X_{2} \rightarrow X_{1}^{\mathbf{t}}\right],\left[X_{2}^{\mathbf{f}} \longmapsto X_{3} \rightarrow X_{2}^{\mathbf{t}}\right]\right) .
$$

with $\operatorname{deg} X_{1}^{\mathbf{t}}=d-l_{1}, \operatorname{deg} X_{2}^{\mathbf{t}}=d-l_{2}$ and $\operatorname{deg} X_{3}^{\mathbf{t}}<d-l_{2}$. Since

$$
\stackrel{\circ}{R}_{d, l_{3}, l_{2}, l_{1}}=\stackrel{\circ}{Y}_{d, l_{3}, l_{2}, l_{1}}=E_{d, l_{2}, l_{1}}^{l_{2}} \cap \stackrel{\circ}{Z}_{d, l_{3}}^{l_{2}},
$$


by the definition of $\stackrel{\circ}{Y}_{d, l_{3}, l_{2}, l_{1}}, E_{d, l_{2}, l_{1}}^{l_{2}} \cap{\stackrel{\circ}{Z_{d, l_{3}}}}_{l_{2}}$ parametrizes such sequences with $\operatorname{deg} X_{1}^{\mathbf{t}}=d-l_{1}, \operatorname{deg} X_{2}^{\mathbf{t}}=d-l_{2}$ and $\operatorname{deg} X_{3}^{\mathbf{t}}=d-l_{3}$.

Continuing this argument, we eventually obtain the parameter-space interpretation for $E_{d, \sigma}^{l_{m}}: E_{d, \sigma}^{l_{m}}$ parametrizes sequences of the form

$$
\left(\left[V_{\mathbb{P}^{1}} \rightarrow X_{1}\right],\left[X_{1}^{\mathbf{f}} \longmapsto X_{2} \rightarrow X_{1}^{\mathbf{t}}\right], \cdots,\left[X_{m}^{\mathbf{f}} \longmapsto X_{m+1} \rightarrow X_{m}^{\mathbf{t}}\right]\right)
$$

with $\operatorname{deg} X_{i}^{\mathbf{t}}=d-l_{i},(i=1, \cdots, m)$ and $\operatorname{deg} X_{m+1}^{\mathbf{t}}<d-l_{m}$. For any $r$ with $d>r>l_{m}, E_{d, \sigma}^{l_{m}} \cap Z_{d, r}^{l_{m}}$ parametrizes such sequences with $\operatorname{deg} X_{i}^{\mathrm{t}}=d-l_{i}$, $(i=1, \cdots, m)$ and $\operatorname{deg} X_{m+1}^{\mathbf{t}}=d-r$.

Next, we deal with the modular interpretation of $E_{d, \sigma}^{r}$ for any $r, d>r>l_{m}$. By the lemma before, we have $E_{d, \sigma}^{r}=E_{d, \sigma}^{l_{m}} \backslash Z_{d, r}^{l_{m}}$. Since $Z_{d, r}^{l_{m}}=\bigcup_{i=l_{m}+1}^{r} Z_{d, i}^{l_{m}}$, we know that $E_{d, \sigma}^{l_{m}} \cap Z_{d, r}^{l_{m}}$ parametrizes sequences as above with $\operatorname{deg} X_{i}^{\mathbf{t}}=d-l_{i}$, $(i=1, \cdots, m)$ and $\operatorname{deg} X_{m+1}^{\mathbf{t}} \geq d-r$. Therefore, $E_{d, \sigma}^{r}$, which equals $E_{d, \sigma}^{l_{m}} \backslash Z_{d, r}^{l_{m}}$, parametrizes such sequences with $\operatorname{deg} X_{i}^{\mathbf{t}}=d-l_{i},(i=1, \cdots, m)$ and $\operatorname{deg} X_{m+1}^{\mathbf{t}}<$ $d-r$.

In summary, we have

Proposition 6.4. Let $d>r$. For any $\sigma=\left(l_{m}, \cdots, l_{2}, l_{1}\right) \in \Sigma$ with $l_{m}<r$, the stratum $E_{d, \sigma}^{r}$ of $Q_{d}^{r}$ parametrizes the sequences of the form

$$
\left(\left[V_{\mathbb{P}^{1}} \rightarrow X_{1}\right],\left[X_{1}^{\mathbf{f}} \longmapsto X_{2} \rightarrow X_{1}^{\mathbf{t}}\right], \cdots,\left[X_{m}^{\mathbf{f}} \longmapsto X_{m+1} \rightarrow X_{m}^{\mathbf{t}}\right]\right)
$$

with $\operatorname{deg} X_{i}^{\mathbf{t}}=d-l_{i},(i=1, \cdots, m)$ and $\operatorname{deg} X_{m+1}^{\mathbf{t}}<d-r$.

Corollary 6.5. The boundary $\widetilde{Q}_{d} \backslash \stackrel{Q}{Q}_{d}$ comes equipped with a natural stratification with strata $E_{d, \sigma}$ indexed by $\sigma=\left(l_{m}, \cdots, l_{2}, l_{1}\right)$ with $d>l_{m}>\cdots>l_{1} \geq 0$.. The stratum $E_{d, \sigma}$ parametrizes the sequences of the form

$$
\left(\left[V_{\mathbb{P}^{1}} \rightarrow X_{1}\right],\left[X_{1}^{\mathbf{f}} \longmapsto X_{2} \rightarrow X_{1}^{\mathbf{t}}\right], \cdots,\left[X_{m}^{\mathbf{f}} \longmapsto X_{m+1} \rightarrow X_{m}^{\mathbf{t}}\right]\right)
$$

such that $\operatorname{deg} X_{i}^{\mathbf{t}}=d-l_{i}, i=1, \cdots, m$, and the last sheaf $X_{m+1}$ is the unique one that is locally free.

This proves Theorem 1.3.

\section{REFERENCES}

[HL97] D. Huybrechts and M. Lehn. The Geometry of Moduli Spaces of Sheaves. Vieweg \& Sohn Verlagsgesellschaft mbH, Braunschweig/Wiesbaden, 1997. 
[HLS11] Y. Hu, J. Lin, and Y. Shao, A Compactification of the Space of Algebraic Maps from $\mathbb{P}^{1}$ to $\mathbb{P}^{n}$. Communications in Analysis and Geometry 19 (2011), no. 1, 1-30.

[Shao11] Y. Shao. A compactification of the space of parametrized rational curves in Grassmannians. arXiv:1108.2299

[Str87] S. Strømme. On Parametrized Rational Curves in Grassmann Varieties. Lecture Notes in Mathematics 1266, Springer-Verlag, Berlin, New York, 1987.

[Vain82] I. Vainsencher. Schubert calculus for complete quadrics. In Enumerative Geometry and Classical Algebraic Geometry (Nice 1981), volume 24 of Progress in Mathemtics, pages 199235. Birkhauser, 1982.

[Vain84] I. Vainsencher. Complete collineations and blowing up determinantal ideals. Math. Ann., 267, 1984.

\section{$\mathrm{Yi} \mathrm{Hu}$}

Department of Mathematics

University of Arizona

USA

E-mail: yhu@math.arizona.edu

Yijun Shao

Department of Mathematics University of Arizona

USA

E-mail: yijun.shao@gmail.com 